\title{
Laminins affect $T$ cell trafficking and allograft fate
}

\author{
Kristi J. Warren,1 Daiki Iwami, ${ }^{1}$ Donald G. Harris, ${ }^{2}$ Jonathan S. Bromberg, ${ }^{1,2,3}$ and Bryna E. Burrell ${ }^{1}$ \\ ${ }^{1}$ Center for Vascular and Inflammatory Diseases, ${ }^{2}$ Department of Surgery, and ${ }^{3}$ Department of Microbiology and Immunology, \\ University of Maryland School of Medicine, Baltimore, Maryland, USA.
}

\begin{abstract}
Lymph nodes (LNs) are integral sites for the generation of immune tolerance, migration of $\mathrm{CD}^{+} \mathrm{T}^{\mathrm{T}}$ cells, and induction of Tregs. Despite the importance of LNs in regulation of inflammatory responses, the LN-specific factors that regulate $T$ cell migration and the precise $L N$ structural domains in which differentiation occurs remain undefined. Using intravital and fluorescent microscopy, we found that alloreactive $T$ cells traffic distinctly into the tolerant $\mathrm{LN}$ and colocalize in exclusive regions with alloantigen-presenting cells, a process required for Treg induction. Extracellular matrix proteins, including those of the laminin family, formed regions within the $\mathrm{LN}$ that were permissive for colocalization of alloantigen-presenting cells, alloreactive $T$ cells, and Tregs. We identified unique expression patterns of laminin proteins in high endothelial venule basement membranes and the cortical ridge that correlated with alloantigen-specific immunity or immune tolerance. The ratio of laminin $\alpha 4$ to laminin $\alpha 5$ was greater in domains within tolerant LNs, compared with immune LNs, and blocking laminin $\alpha 4$ function or inducing laminin $\alpha 5$ overexpression disrupted T cell and DC localization and transmigration through tolerant LNs. Furthermore, reducing $\alpha 4$ laminin circumvented tolerance induction and induced cardiac allograft inflammation and rejection in murine models. This work identifies laminins as potential targets for immune modulation.
\end{abstract}

\section{Introduction}

Lymph nodes (LNs) are secondary lymphoid organs that serve as integral sites for the control of immunity and tolerance. These encapsulated organs consist of a stromal reticular network that forms the framework for the outermost cortex, middle paracortex, and innermost medulla $(1,2)$. B cells, follicular dendritic cells, and macrophages reside in the follicles of the cortex. In the middle paracortex, the T cells, fibroblastic reticular cells (FRCs), and dendritic cells (DCs) reside in the T cell zone. The innermost medullary layer contains the lymphatic medullary cords, lined by lymphatic endothelial cells and separated by the medullary sinuses.

Appropriate leukocyte trafficking is necessary for the induction of alloantigen-specific tolerance (3-8). Tregs migrate through the allograft, where they locally suppress alloantigen acquisition by inflammatory DCs. Tregs then migrate to the LNs, where they suppress alloantigen-specific $\mathrm{CD}^{+} \mathrm{T}$ cell priming $(5,7-11)$. Tolerance-inducing plasmacytoid DCs ( $\mathrm{pDCs}$ ) also circulate through the allograft, acquiring antigen and transporting it to the LNs, where they induce antigen-specific Treg differentiation $(3-5,12)$. Within the LNs, alloantigen-presenting pDCs and Tregs associate with the high endothelial venules (HEVs) in the cortical ridge (CR), exposing naive alloreactive cells to alloantigen and regulation almost immediately upon LN entry $(3,13-15)$. The timing of alloantigen presentation to alloreactive $\mathrm{CD} 4^{+} \mathrm{T}$ cells is important to their fate, as alloreactive cells that are present at the induction of tolerance become transiently activated and differentiate into Tregs, whereas naive alloreactive cells transferred at later times

Conflict of interest: At the time of manuscript submission, Kristi J. Warren, Daiki Iwami, Donald G. Harris, Jonathan S. Bromberg, and Bryna E. Burrell were employed by the University of Maryland School of Medicine, which has filed a patent application pertaining to the data herein.

Citation for this article: J Clin Invest. 2014;124(5):2204-2218. doi:10.1172/JCI73683 after initiation of tolerization become anergic and apoptotic (4). The colocalization of naive alloreactive cells with Tregs, alloantigen, and pDCs within the LNs is integral to the induction of allograft tolerance, although the mechanisms regulating these movements are not known.

$\mathrm{T}$ cells enter the LNs via blood through the HEVs in the paracortex (16). These specialized vessels are lined abluminally with basement membrane stromal fibers. HEVs are luminally lined with blood endothelial cells (BECs) expressing the CD62L ligand peripheral node addressin (PNAd), which mediates the tethering and rolling of T cells $(5,17)$. T cell arrest on the endothelium is mediated by CCR7 and CXCR4 recognition of CCL21 and CXCL12, respectively, and these chemokines decorate the luminal surface of the HEV. These interactions result in the upregulation of $\mathrm{T}$ cell integrins that allow for the arrest of T cells within the HEV. Lymphocytes then migrate either between or through endothelial cells before crossing the HEV basement membrane to the abluminal side. Pockets form between the endothelial cells and basement membrane fibers and serve as a malleable checkpoint structure that controls LN cellularity (18). Following HEV extravasation, T cells remain in the abluminal perivascular space. They then interact with a CCL19 and CCL21 gradient and migrate along stromal fibers produced by and intertwined with FRCs toward the T cell zone (16). The regulation of the checkpoints into, between, and beyond the HEV endothelial cells and basement membrane is poorly understood.

LN structure is integral to the generation of an appropriate immune response (19-21). Lymphoid tissue remodeling (22-25), and remodeling of the HEVs themselves $(26,27)$, are common themes following immune challenge. The stromal fibers ER-TR7 $(14,28,29)$ and laminin $(30,31)$ are made by a variety of cell types and form both HEV basement membranes and the LN reticular network. Lymphocytes induce FRCs to produce ER-TR7 in the CR, a region of the paracortex between the $\mathrm{T}$ and $\mathrm{B}$ cell zones through 
A
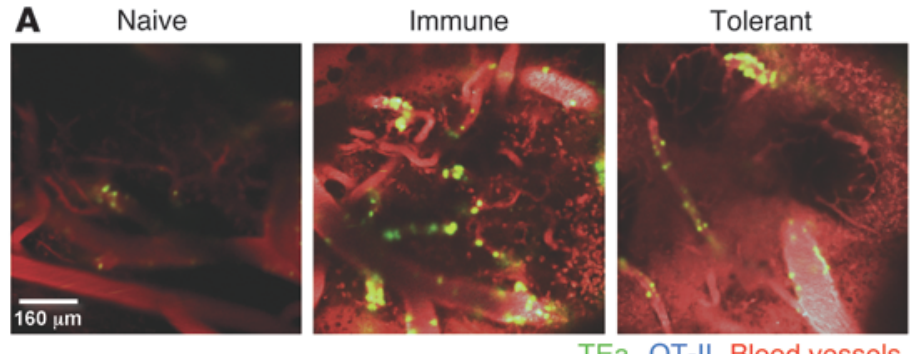

TEa OT-II Blood vessels

B

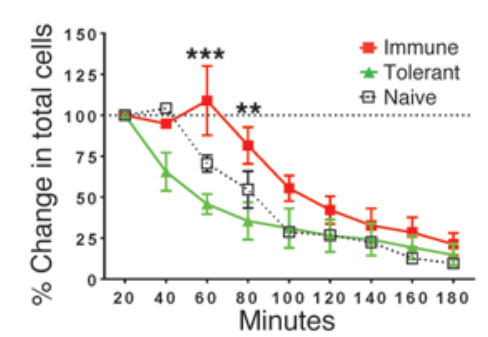

Total cells

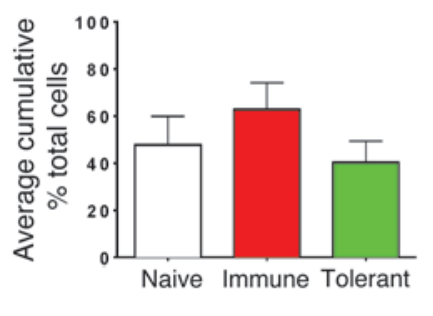

Circulating
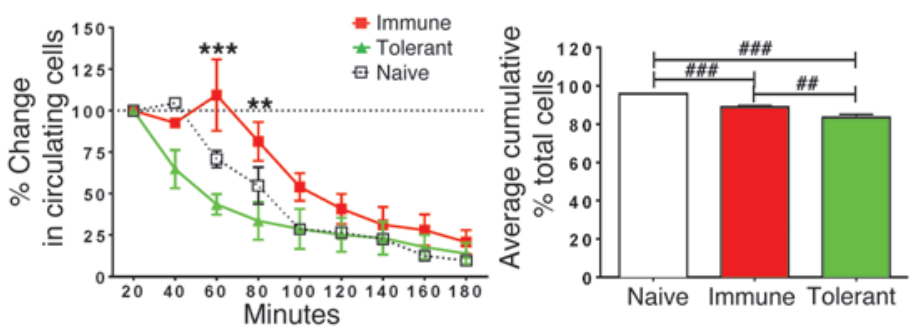

D

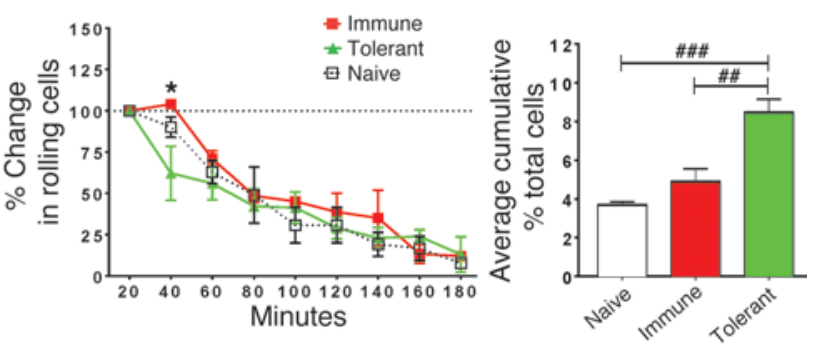

$\mathbf{E}$

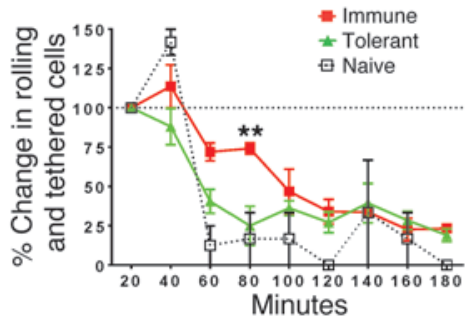

$\mathbf{F}$

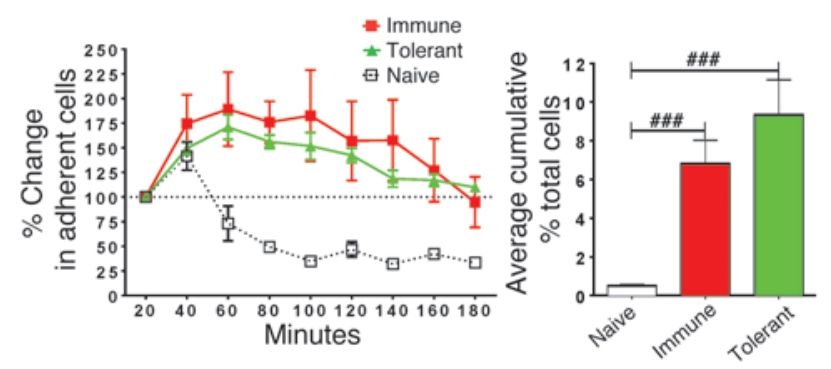

Figure 1

Distinct trafficking patterns of antigen-specific CD4+ $\mathrm{T}$ cells through HEVs following the induction of immunity and tolerance. CD4+ TEa cells and CD4+ OT-II cells were isolated, differentially labeled, and transferred at the time of DST with or without anti-CD40L injection to naive (untreated, $n=2$ ), immune (DST, $n=3$ ), or tolerized (DST + anti-CD40L, $n=3$ ) C57BL/6 mice. Dextran revealed blood vessels. Total cells were categorized. Inguinal LNs imaged 20 to 180 minutes after cell transfer, on a single plane, for 800 frames ( 12 minutes). (A) Representative images of inguinal LNs; original magnification, $\times 200$. (B) Total number of cells detected 20 minutes after cell transfer quantified and set as $100 \%$ (horizontal dashed line). The percent increase or decrease of the total number was calculated for each time point. Bars represent the average cumulative percent change per group from 20 to 180 minutes after cell transfer. (C-F) Left column, line graphs: The number of cells circulating (C), rolling (D), rolling and tethered $(E)$, or adherent $(\mathbf{F})$ detected at 20 minutes after cell transfer set as $100 \%$. Percent change calculated for each time point. Right column: Bars represent the average cumulative percent of total cells detected per group from 20 to 180 minutes after cell transfer. Data presented

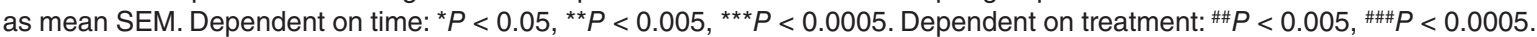

which T cells enter the LN via HEVs $(14,28,29)$. The CR forms a structural scaffolding seeded by DCs; this region is integral to bringing T cells and antigen-presenting cells (APCs) together (14). ER-TR7 fibers and FRCs encase the HEV, where they both aid in maintaining the HEV basement membrane and controlling $\mathrm{T}$ cell travel from the HEV into the LN parenchyma (20). The mechanisms regulating fiber structure and remodeling are incompletely defined.

The laminins are a family of heterotrimeric glycoproteins with a variety of adhesive and stimulatory functions. Their $\alpha$ chains contain integrin-binding sites and therefore affect cell migration. Two isoforms are particularly associated with $\mathrm{LN}$ and blood vessel structure and function $(30,31)$. Produced by vascular endothelial cells and lymphocytes, laminin-411 is an endothelial basement membrane protein essential for the maturation and structural integrity of microvessels (32). Laminin-411 contains the laminin $\alpha 4$ chain and promotes $T$ cell migration (33). Laminin-511 and laminin-521 are also present in the endothelial basement membrane (34) and are produced early during embryogenesis (35). Laminin-511 contains the laminin $\alpha 5$ chain and fails to promote $\mathrm{T}$ cell migration, although $\mathrm{T}$ cell costimulatory properties have been reported (33). Recent work suggests the presence and ratio of these laminin isoforms may dictate inflammatory responses and maturational niches for $\mathrm{T}$ and $\mathrm{B}$ cells $(36,37)$, although how they are regulated has not been investigated.

We investigated the dynamics of $\mathrm{CD}^{+} \mathrm{T}$ cell entry into LNs, LN structural remodeling, and their relation to $\mathrm{T}$ cell fate. We found that, following the induction of tolerance versus immunity, $T$ cells differentially enter the LN via the HEVs and localize in distinct domains in the LN. These dynamics are established acutely within minutes of immune stimulation. Movements of CD4 ${ }^{+} \mathrm{T}$ cells, Tregs, and alloantigen-specific pDCs are dictated by the stromal fiber components laminin $\alpha 4$ and laminin $\alpha 5$. Manipulation or inhibition of 
A

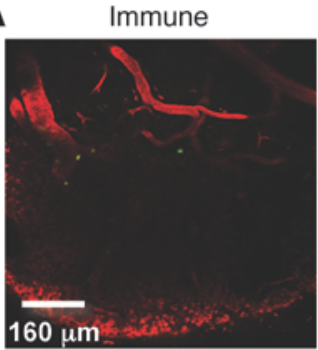

Tolerant

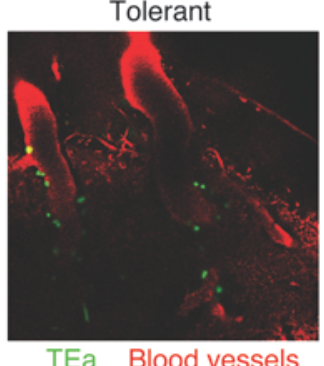

B

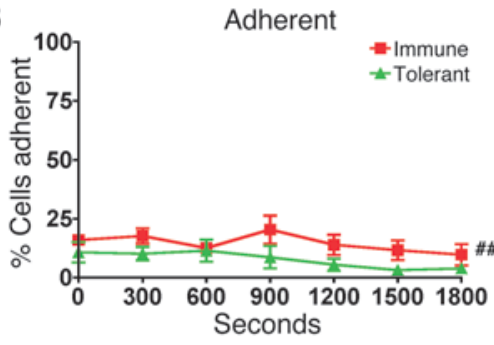

C

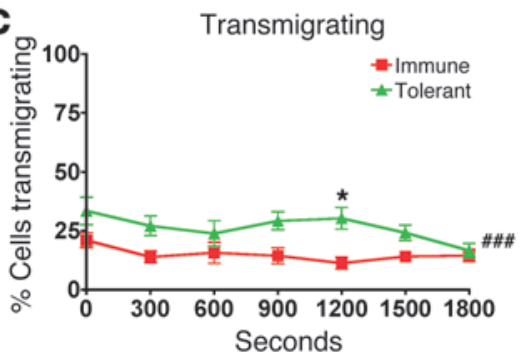

D

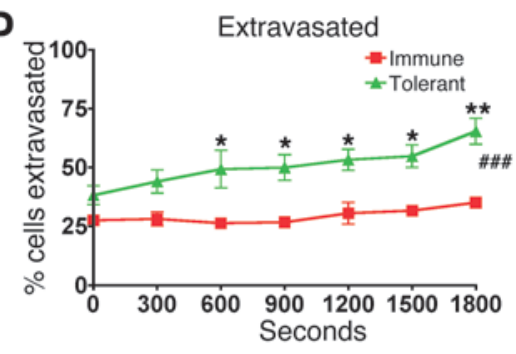

$\mathbf{E}$

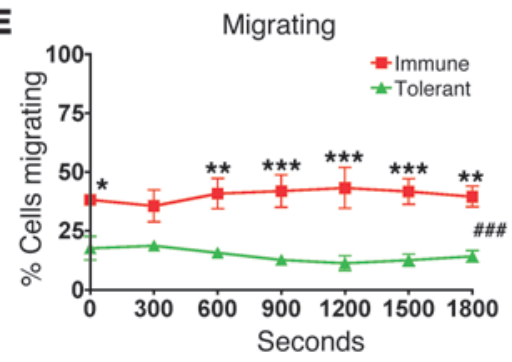

Figure 2

Distinct trafficking patterns of antigen-specific CD4+ $T$ cells through $L N s$ following the induction of immunity and tolerance. (A) Representative images of inguinal LNs, 4 hours after cell transfer; original magnification, $\times 200$. (B-E) Percent of total number of cells detected per time point, starting 3.5 hours after cell transfer, that were adherent (B), transmigrating (C), extravasated (D), or migrating (E). Data presented as mean \pm SEM.

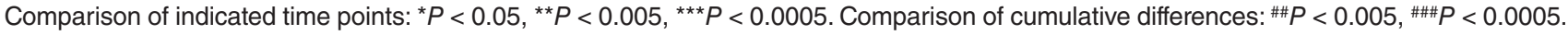

fiber expression or function alters the localization of these cells and acts as a molecular switch for tolerance and immunity.

\section{Results}

Distinct trafficking of antigen-specific $C D 4^{+} T$ cells to tolerant LNs. Multiphoton microscopy was used to image antigen-specific cells as they entered the LN vasculature and interacted with the HEVs during the induction of alloantigen immunity or tolerance. C57BL/6 mice were made immune via administration of donor-specific transfusion (DST; $10^{7} \mathrm{BALB} / \mathrm{c}$ splenocytes) or tolerant via administration of DST plus anti-CD $40 \mathrm{~L} \mathrm{mAb}(250 \mu \mathrm{g} /$ dose $)$. This protocol of tolerance induction by use of DST in the context of costimulatory blockade is well established and widely used (3-5, 38-46). Simultaneously with DST with or without anti-CD40L administration, $\mathrm{CD}^{+} \mathrm{T}$ cells that recognize $\mathrm{BALB} / \mathrm{c}$ donor $\mathrm{I}-\mathrm{E}^{\mathrm{d}}$ antigen presented by $\mathrm{C} 57 \mathrm{BL} / 6$ recipient I-A $A^{b}$ from TEa TCR transgenic mice were labeled with CFSE and adoptively transferred. To evaluate antigen specificity, antigen-irrelevant OT-II TCR Tg CD4 ${ }^{+}$cells specific for ovalbumin were labeled with Qtracker 655 and cotransferred. TRITC-labeled 70-kDa dextran was administered to visualize blood vessels. Mice were imaged for 800 frames (about 12 minutes), every 20 minutes, from 20 to 180 minutes after cell transfer (Figure 1, A-F, Supplemental Videos 1-6, and Supplemental Figure 1A; supplemental material available online with this article; doi:10.1172/JCI73683DS1). Accumulation of $\mathrm{CD}^{+} \mathrm{TEa}$ antigen-specific cells within the $\mathrm{LN}$ vasculature was initially visualized within 20 minutes of cell transfer and antigen exposure; however, the majority of cells were in the circulation at that time point. Over time, antigen-specific TEa T cells accumulated and adhered to the vasculature, while antigen-irrelevant OT-II T cells did not (Supplemental Figure 1B).

The total number of $\mathrm{CD}^{+}$TEa cells detected in the $\mathrm{LN}$ vasculature and HEVs over the entire 20-to-180-minute imaging timeframe was quantified (Figure 1B). The total number of cells detected in naive and immune mice initially increased but returned to baseline levels by 60 minutes after cell transfer. In contrast, the total number of cells detected in tolerant LNs decreased immediately and continued to decrease throughout the experiment (Figure 1B). Although these differences failed to reach significance, these trends were also apparent when the cumulative change in the number of cells observed over the total 3-hour time frame was quantified (Figure 1B). These results showed that in naive and immune mice, antigen-specific cells were present at higher numbers, whereas in tolerant mice, antigen-specific cells progressively failed to be detected in the LN vasculature and on the HEVs. The detection of fewer cells in tolerant mice was likely not due to anti-CD40L mAb directly depleting activated alloantigen-reactive cells $(47,48)$, as we and others have demonstrated anti-CD40L to modulate the differentiation of, but not acutely deplete, activated alloreactive T cells $(4,49,50)$.

To quantify the changes in cell movements in the $\mathrm{LN}$ vasculature and HEVs, cells were assigned to 1 of 4 groups to categorize their migratory activities as follows: (a) circulating (cells present for 1 frame only), (b) rolling (cells present for 2-6 frames before being released into circulation), (c) rolling and tethered (cells rolled along the luminal surface of the HEV and adhered during imaging), and (d) adherent (cells that remained stagnant for the entirety of imaging time). Imaging of naive and immune LNs revealed an initial increase in the number of cells in the circulation and rolling in blood vessels, while the number of cells in circulation and rolling in the tolerant LNs progressively decreased (Figure 1, C and D). Over 3 hours, cumulatively $95.8 \%$ of detected cells remained in the circulation in the naive LNs, and this percentage was reduced to $88.9 \%$ and $83.4 \%$ in immune and tolerant LNs, respectively $(P<0.0005$, naive vs. immune/tolerant; $P<0.005$, immune vs. tolerant; Figure $1 \mathrm{C})$. 
A

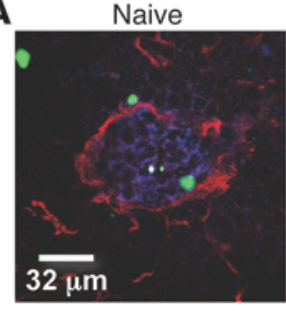

TEa ER-TR7 PNAd

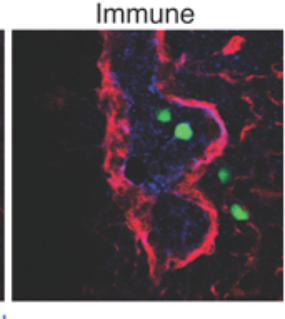

Immune

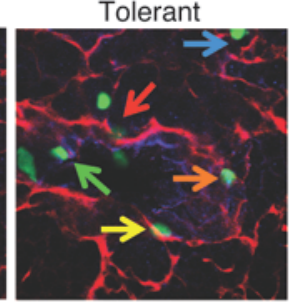

Intraendothelial, surrounded by PNAd

Endothelial/basement membrane, surrounded by PNAd + ER-TR7

Intra-basement membrane, surrounded by ER-TR7

Abluminal surface HEV, touching HEV

In the cortical ridge, not touching HEV

Not in cortical ridge, in lymph node parenchyma

B

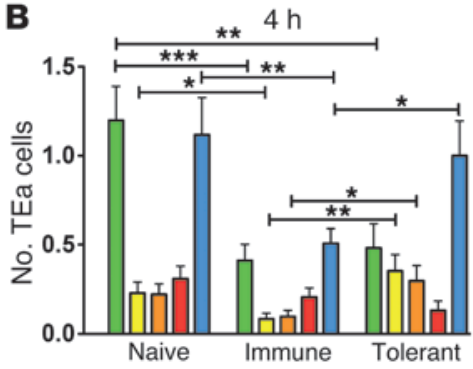

C

$24 \mathrm{~h}$

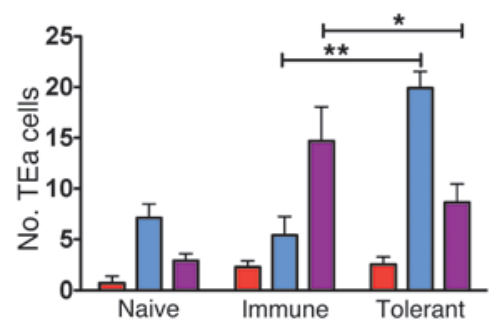

Figure 3

Antigen-specific $C D 4^{+} T$ cells home to the cortical ridge following the induction of tolerance. CD4 + TEa cells were labeled with CFSE and transferred to naive, immune (DST), or tolerized (DST + anti-CD40L) C57BL/6 mice. Recipients were euthanized 4 hours (B) or 24 hours (C) after cell transfer. LNs were harvested, and 5 - $\mu \mathrm{m}$ cryosections were analyzed for cell migration and location by fluorescent immunohistochemistry of structures identified by ER-TR7 ${ }^{+}$stromal fibers and PNAd+ HEVs. Cells were categorized as intraendothelial, within endothelium/basement membrane, within basement membrane, outside HEV, within cortical ridge, and beyond cortical ridge. (A) Representative fluorescent confocal immunohistochemistry of antigen-specific CD4 ${ }^{+} \mathrm{T}$ cells. Arrows denote cells present in designated locations. Original magnification, $\times 1,000$ (leftmost 3 panels), $\times 100$ (right panel). (B and C) Quantitation of T cell distribution 4 hours after cell transfer from $\times 1,000$ images $(\mathbf{B})$ and 24 hours after cell transfer from $\times 100$ images (C). Data presented as mean \pm SEM. $n=3-5$ mice per treatment; $1-4$ LNs per mouse; ${ }^{\star} P<0.05,{ }^{* \star} P<0.005,{ }^{\star \star *} P<0.0005$.

Cumulatively, rolling cells accounted for $3.7 \%$ of cells in naive LNs, compared with $4.9 \%$ and $8.5 \%$ of cells in immune and tolerant LNs, respectively $(P<0.0005$, naive vs. tolerant; $P<0.005$ immune vs. tolerant; Figure 1D). The number of cells rolling and tethered to the blood vessels initially increased in naive and immune LNs and steadily decreased in tolerant LNs (Figure 1E). Cumulatively, $0.05 \%$ of cells in naive LNs, $0.69 \%$ of cells in immune LNs, and $0.82 \%$ of cells in tolerant LNs were rolling and tethered to the vascular endothelium $(P<0.0005$, naive vs. immune/tolerant; Figure 1E). The percentage of cells adherent to the vascular endothelium in naive LNs transiently increased, and in immune and tolerant LNs increased from 20 to 60 minutes, plateaued from 60 to 100 minutes, and returned to baseline levels by 180 minutes after cell transfer (Figure $1 \mathrm{~F})$. Cumulatively, $0.49 \%$ of cells in naive LNs, $6.8 \%$ of cells in immune LNs, and $9.3 \%$ of cells in tolerant LNs were adherent to the vascular endothelium $(P<0.0005$, naive vs. immune/tolerant; $P<0.05$, immune vs. tolerant; Figure $1 \mathrm{~F})$.

These data showed that antigen-specific cells in naive mice remained in circulation, with few cells rolling, rolling and tethered, or adhering to the vascular endothelial bed in the LNs. Antigenspecific cells in immune mice largely remained in circulation, although some interacted with the vascular endothelium to the lumen with blood around all sides of the cell), (b) transmigrating (between the endothelial cells and the basement membrane of the HEV), (c) extravasated (lining the abluminal basement membrane of the HEV), and (d) migrating (cells that have left the HEV and traveled into the LN parenchyma; Figure 2, A-E). At individual time points, the percentage of detected cells that were adherent to the vasculature were similar in immune and tolerant LNs. Cumulatively, during the 30 minutes of observation, adherent cells accounted for $14.6 \%$ and $7.7 \%$ of the total cells detected in immune and tolerant LNs, respectively $(P<0.005$, immune vs. tolerant; Figure 2B). An increased cumulative percentage of cells was observed transmigrating in tolerant HEVs in comparison with immune HEVs (26.3\% vs. $14.9 \%$, respectively; $P<0.0005$, immune vs. tolerant; Figure 2C). An increased percentage of cells that had extravasated through the HEV, yet remained adherent to the abluminal side of the vascular wall, was also observed in tolerant versus immune LNs, from 600 to 1,800 seconds of the observation period, and cumulatively $(50.7 \%$ vs. $29.5 \%$, respectively; $P<0.0005$, immune vs. tolerant; Figure 2D). Relative to cells in immune LNs, significantly fewer cells migrated away from tolerant HEVs and into the LN parenchyma at each time point observed, and cumulatively $(14.6 \%$ in tolerant LNs, $40.1 \%$ in immune LNs; $P<0.0005$, 
A

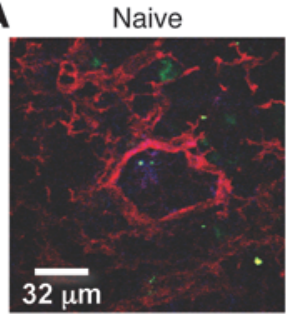

Foxp3 ER-TR7 PNAd

Intraendothelial, surrounded by PNAd

Endothelial/basement membrane, surrounded by PNAd + ER-TR7

Intra-basement membrane, surrounded by ER-TR7

Abluminal surface HEV, touching HEV

In the cortical ridge, not touching HEV

Not in cortical ridge, in lymph node parenchyma

B

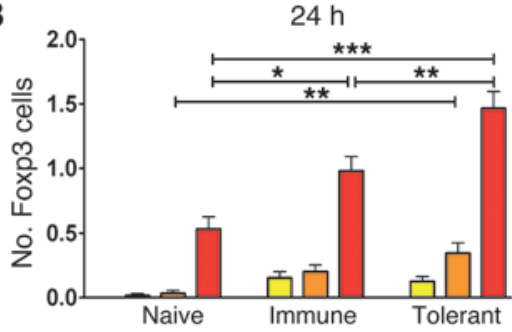

D

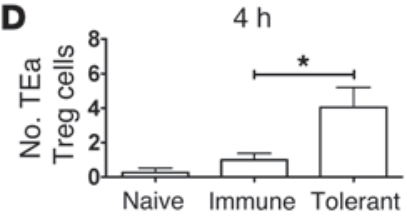

Immune

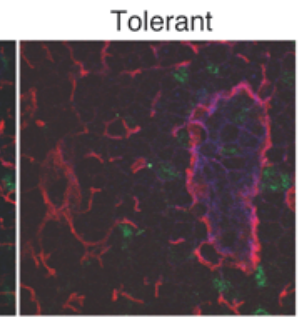

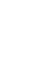

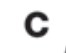

c
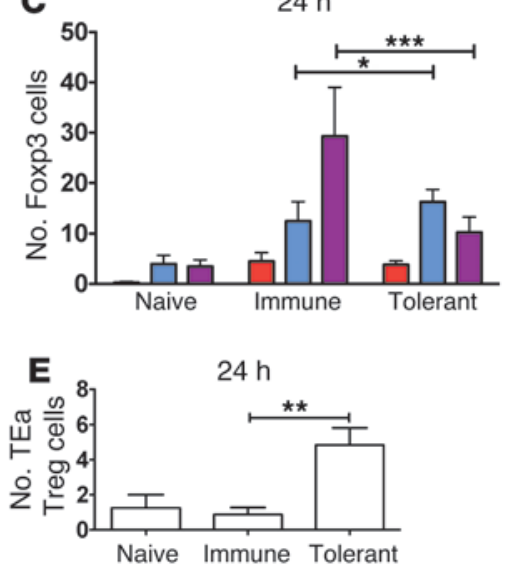

\section{Figure 4}

Regulatory $\mathrm{CD}^{+} \mathrm{T}$ cells home to the cortical ridge following the induction of tolerance. CD4+ TEa cells were labeled with CFSE and transferred to naive, immune (DST), or tolerized (DST + anti-CD40L) C57BL/6 mice. (A) Representative fluorescent confocal immunohistochemistry of Foxp3 $3^{+}$Tregs 24 hours after cell transfer; original magnification, $\times 1,000$. (B and C) Quantitation of Foxp3+ Tregs distribution 24 hours after cell transfer from $\times 1,000$ (B) and $\times 100$ (C) images. (D and E) Distribution of antigen-specific TEaTregs 4 hours (D) and 24 hours (E) after cell transfer from $\times 100$ images. Data presented as mean \pm SEM. $n=3-5$ mice per treatment; $1-4$ LNs per mouse; ${ }^{*} P<0.05,{ }^{* *} P<0.005$, ${ }^{* \star *} P<0.0005$ immune vs. tolerant; Figure 2E). These data showed that antigen-specific cells interacted for an extended time with the HEV in tolerant versus immune or naive LNs. Cells in tolerant LNs were found actively transmigrating or extravasated after becoming adherent to the HEV. In immune LNs, cells were more often detected in the LN parenchyma, suggesting that the few cells that did adhere to the HEV quickly traversed the vascular basement membrane. Taken together, these findings showed that tolerance induction resulted in a more protracted intimate contact between antigen-specific cells and the HEV.

Antigen-specific and regulatory $\mathrm{CD}^{+} \mathrm{T}$ cells bome to the CR following tolerance induction. Since antigen-specific $\mathrm{CD}^{+} \mathrm{T}$ cells acutely entered the LNs and migrated to discrete regions following the induction of immunity versus tolerance, fluorescent confocal immunohistochemistry was used to characterize more precisely their location. Discrete microdomains were defined as (18): (a) intraendothelial (lymphocytes completely surrounded by PNAd ${ }^{+}$ BECs that line the luminal side of the HEV); (b) endothelial/ basement membrane (lymphocytes occupy spaces defined by $\mathrm{PNAd}^{+} \mathrm{BEC}$ on one side and ER-TR7 ${ }^{+}$basement membrane proteins on the other); (c) intra-basement membrane (completely surrounded by the ER-TR $7^{+}$basement membrane); (d) outside HEV (cells in contact with the ER-TR7 ${ }^{+}$HEV basement membrane on the abluminal side of the HEV); (e) in CR (cells present in optical field containing the HEV but not in contact with the abluminal ER-TR7 ${ }^{+}$basement membrane); and (f) beyond the CR (located in the LN parenchyma, beyond the HEV and ER-TR7 ${ }^{+}$dense fiber region; Figure $3 \mathrm{~A}$ ).
Four hours after the induction of immunity or tolerance, differences in the distribution of antigen-specific TEa $T$ cells within and surrounding the HEV were characterized (Figure 3B). In the naive LNs, the majority of cells were either entering the endothelium (intraendothelial) or within the CR. Similarly, in the immune LNs, the majority of cells were entering the endothelium or within the CR and not in contact with the HEV. In contrast, in the HEVs of tolerant LNs, TEa T cells were located primarily between the endothelium and basement membrane (tolerant vs. immune; $P<0.005)$. These findings indicated that antigen-specific TEa $T$ cells followed distinct pathways of migration to enter a tolerant versus immune $\mathrm{LN}$. As a control, ovalbumin-specific CD4 $4^{+} \mathrm{TCR}$ Tg OT-II cells were cotransferred with TEa cells to tolerant and immune mice and their locations determined. The distributions of these antigen-irrelevant $\mathrm{CD}^{+}$cells were similar between tolerant and immune HEVs and mirrored the distributions observed with TEa cells in naive LNs (Supplemental Figure 2).

We next examined the distribution of TEa T cells across the various regions of the entire $\mathrm{LN}$ (in contact with $\mathrm{HEV}$, in CR yet not touching HEV, or not in CR) 24 hours after tolerance induction (Figure $3 \mathrm{C}$ ). The majority of TEa T cells resided in the $\mathrm{CR}$ after tolerance induction $(P<0.005$ vs. immune). In contrast, there was an increase in TEa T cells located outside the CR in the immune LNs $(P<0.05$ vs. tolerant). These findings further supported the notion that antigen-specific $\mathrm{T}$ cells had distinct pathways of migration into the $\mathrm{LN}$, as well as designated areas within the LN where they resided, potentially affecting the outcome of the immune response. 
A

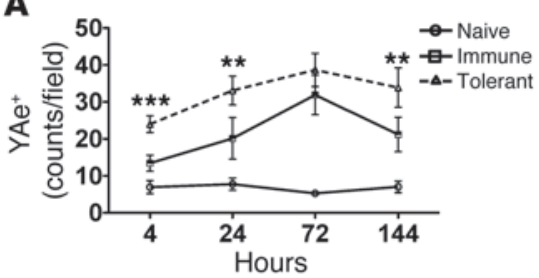

C

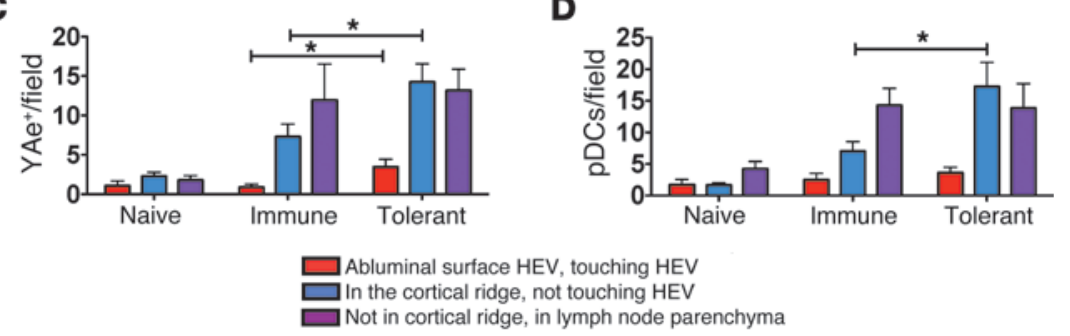

B

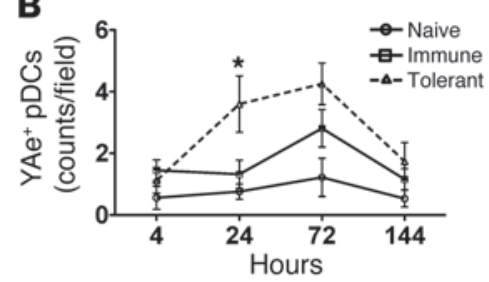

D

\section{Figure 5}

Alloantigen-presenting pDCs migrate to the cortical ridge after tolerance induction. Fluorescent immunohistochemistry of LN cryosections taken from immune (DST) or tolerant (DST + anti-CD40L) mice, 4 hours to 6 days after treatment. (A and $\mathbf{B}$ ) LNs were imaged in their entirety for total number of alloantigen-presenting $\mathrm{YAe}^{+}$cells $(\mathbf{A})$ and alloantigen-presenting YAe+PDCA-1+ pDCs (B) quantified across each LN cryosection. (C and $\mathbf{D})$ The distribution of $\mathrm{YAe}^{+}$cells (C) and pDCs (D) enumerated as cells outside the HEV, in the cortical ridge, and beyond the cortical ridge 24 hours after treatment. Images acquired at $\times 100$. Data presented as mean \pm SEM. $n=4$ mice per group; $1-4$ LNs per mouse; ${ }^{\star} P<0.05,{ }^{* *} P<0.005,{ }^{* \star *} P<0.0005$.
We previously demonstrated accumulation and differentiation of naive antigen-specific cells to induced Tregs (iTregs) within the CR of tolerant mice (4). The observed differences in antigen-specific cell migration to and location in the HEV and CR may correlate with differences in cell fate. Therefore, Foxp $3^{+}$cells were quantified, and an increased number was noted on the abluminal surface of the HEV 24 hours after the induction of tolerance $(P<0.005$ vs. immune; $P<0.0005$ vs. naive; Figure 4 , A and B). Furthermore, Foxp $3^{+}$Tregs accumulated in the CR following the induction of tolerance, whereas immunity was associated with an accumulation of Foxp $3^{+}$Tregs located in the non-CR region (Figure 4C). TEa mice possess very few natural Tregs $(4,51)$. As a result, detected TEa Foxp $3^{+}$Tregs must by definition be iTregs. The number of alloantigen-specific iTregs ( $\mathrm{TEa}^{+} \mathrm{Foxp}^{+}$, TEaTregs) was quantified in the CR. Four hours after the induction of tolerance, more TEaTregs were in the CR of the tolerant LNs $(P<0.05$ vs. immune; Figure 4D). The induction and accumulation of TEaTregs in the CR of the tolerant LNs persisted for at least 24 hours after treatment $(P<0.005$, immune vs. tolerant; Figure 4E). Taken together, these findings demonstrated that following tolerance induction, antigen-specific cells and Foxp $3^{+}$Tregs migrated to and accumulated within and around the HEV and CR, and thereafter a subset of these cells differentiated into antigen-specific iTregs.

Specific alloantigen-presenting $p D C$ s migrate to the CR after tolerance induction. We previously demonstrated that specific alloantigen-presenting pDCs accumulated in the CR of tolerant LNs following cardiac allograft transplantation (3). We next determined the total number of alloantigen-presenting cells detected by the YAe antibody (recognizes I-E ${ }^{\mathrm{d}}$ presented by I-A ${ }^{\mathrm{b}} ; \mathrm{YAe}^{+}$; Figure 5A) and tolerance-inducing alloantigen-presenting $\mathrm{pDCs}\left(\mathrm{YAe}^{+} \mathrm{pDCs}\right.$; Figure 5B) located in LNs 4 hours to 6 days after the induction of immunity or tolerance. In the tolerant LNs, the number of YAe ${ }^{+}$ cells was significantly greater within 4 hours of treatment, and $\mathrm{YAe}^{+}$cell numbers persisted over time as compared with those in the immune LNs. A similar change in $\mathrm{YAe}^{+} \mathrm{pDCs}$ in the tolerant LNs was observed, with a significant increase in their numbers by 24 hours compared with those in immune or naive LNs $(P<0.05)$. YAe ${ }^{+}$cells in contact with HEVs or in the CR were increased following tolerance induction (Figure $5 \mathrm{C}$ ). In addition, the total number of pDCs in the CR of the tolerant LNs was significantly higher $(P<0.05$; Figure 5D). These results showed that alloantigen presentation in the tolerant LNs was distinct in comparison with the immune LNs; during tolerization alloantigen-presenting pDCs, alloantigen-specific $\mathrm{CD}^{+}{ }^{+} \mathrm{T}$ cells, and alloantigen-specific iTregs migrated to and/or accumulated in the same areas within or near the HEV and CR.

Unique LN structural changes occur following the induction of immunity versus tolerance. Differences in the migration and location of $\mathrm{T}$ cells and pDCs associated with HEVs and the CR were evident 4 to 24 hours after the induction of immunity versus tolerance. Stromal fibers regulate lymphocyte movement through both the HEV and the CR and signify structural changes associated with LN remodeling. ER-TR7 identifies structural components of the $\mathrm{LN}$, and laminins dictate lymphocyte movement and activation within the LN $(14,28-31)$. We hypothesized that changes in stromal components dictated the distinct movements and location of pDCs, $\mathrm{CD}^{+} \mathrm{T}$ cells, and Tregs. The total amounts of ER-TR7 and laminin were quantitated by fluorescent immunohistochemistry 24 hours after the induction of tolerance or immunity. After tolerance induction, the percentage of the tolerant LNs positive for ER-TR7 increased significantly compared with immune LNs $(P<0.05$; Figure $6 \mathrm{~A})$. The complexity of ER-TR $7^{+}$fibers branching around the HEV was further quantified, and tolerant HEV branching was significantly increased compared with both naive and immune HEVs $(P<0.0005$, immune vs. tolerant; Figure 6B). Conversely, the percentage of the LNs positive for total laminin increased significantly after the induction of immunity versus tolerance $(P<0.005$; Figure 6C). Together these findings showed that LN stromal fiber remodeling specific to the induction of tolerance versus immunity occurred rapidly after immune stimulation.

Laminin function is largely determined by $\alpha$ chain expression. Laminins containing the $\alpha 4$ and $\alpha 5$ chains have been implicated in lymphocyte migration, egress from blood vessels, and $\mathrm{T}$ cell costimulation; and these laminins are particularly abundant and prominent in the LN compared with other laminin $\alpha$ chains (32, $36,52)$. The expression of laminin $\alpha 4$ and $\alpha 5$ chains in the CR was assessed (Figure 7A). The total area of laminin $\alpha 4$ was increased in the CR of tolerant LNs compared with naive and immune LNs. Laminin $\alpha 4$ expression in the CR of tolerant LNs was significantly different from that in the CR of immune LNs at 24 hours and 


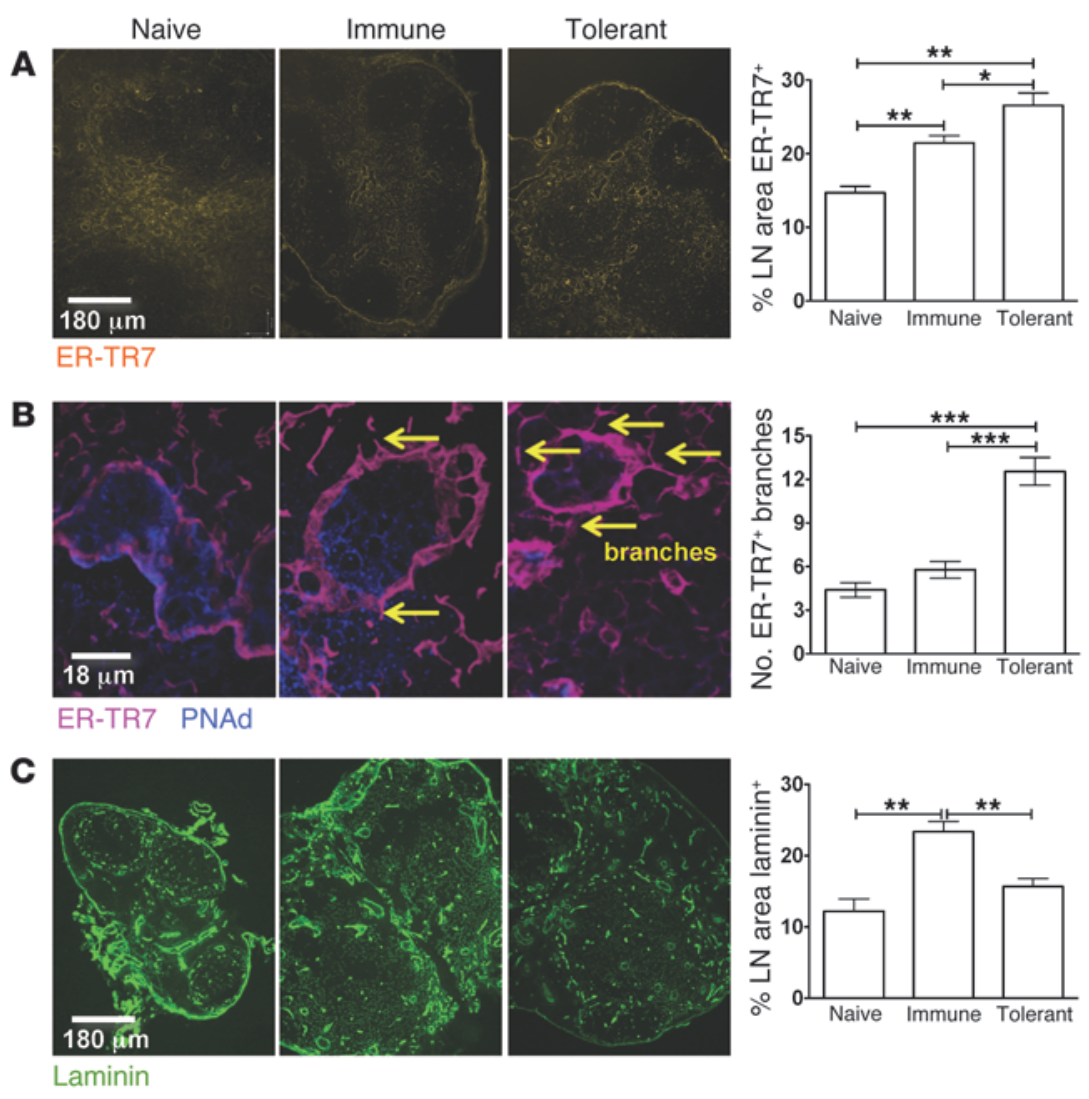

Figure 6

Unique LN structural changes occur following induction of immunity versus tolerance. Mice were made immune or tolerant, 24 hours after treatment; LN cryosections analyzed for total LNs staining positive for ER-TR7 (original magnification, $\times 100$; A), number of ER-TR7 ${ }^{+}$ branches protruding from $\mathrm{PNAd}^{+} \mathrm{HEVs}$ (original magnification, $\times 1,000 ; \mathbf{B}$ ), or total laminin proteins (original magnification, $\times 100 ; \mathbf{C})$. (A and C) $n=3-5$ mice per treatment; $1-4 \mathrm{LNs}$ per mouse $(\times 100)$. (B) $n=3$ mice per treatment; $1-4$ LNs per mouse; $30-100 \mathrm{HEVs}$ per LN $(\times 1,000) .{ }^{\star} P<0.05,{ }^{\star \star} P<0.005,{ }^{\star \star \star} P<0.0005$.

6 days and was different from that in naive LNs at all times (Figure 7B). Conversely, the amount of laminin $\alpha 5$ expressed in tolerant and immune CRs increased similarly, peaking 72 hours after treatment (Figure 7B). The ratios of laminin $\alpha 4$ to laminin $\alpha 5$ in the $\mathrm{CR}$ were also higher in the tolerant group (Figure 7C).

The expression of laminin $\alpha 4$ and laminin $\alpha 5$ associated with the HEV was also measured. There was an increase in laminin $\alpha 5$ in the immune HEV, a relative decrease in laminin $\alpha 4$ in the immune versus the tolerant $\mathrm{HEV}$, and a large difference in the ratio of the fibers between immune and tolerant HEVs $(P<0.05$; Figure 7D). These findings suggested that increased laminin $\alpha 4$ relative to laminin $\alpha 5$ was associated with the induction of tolerance, and increased laminin $\alpha 5$ relative to laminin $\alpha 4$ was associated with immunity.

Laminin $\alpha 4$ and laminin $\alpha 5$ differentially modulate $C D 4^{+} T$ cell motility and transendothelial migration. The migration and location of $\mathrm{CD}^{+} \mathrm{T}$ cells, Foxp $3^{+}$Tregs, and $\mathrm{pDCs}$ in LNs were unique and indicative of tolerance, and these cell movements were accompanied by unique changes in LN structure. The contributions of laminin $\alpha 4$ and laminin $\alpha 5$, alone and in combination, to $T$ cell migration were assessed in a variety of assays that measured random, chemokine-driven, and chemokine-plus-shear-driven migra- tion of $\mathrm{CD}^{+} \mathrm{T}$ cells across vascular endothelial cells. Transwell inserts were coated with lami$\operatorname{nin} \alpha 4$, laminin $\alpha 5$, or laminin $\alpha 4$ plus laminin $\alpha 5$ at different ratios before addition of MS-1 vascular endothelial cells on top of the fibers. This assembly was used to model the laminins constituting the abluminal basement membrane of the HEV. Enriched CD4 ${ }^{+} \mathrm{T}$ cells were added to the top chamber and migrated toward CCL21 in the bottom chamber, and migration characteristics were quantified.

High concentrations of laminin $\alpha 5$ on the abluminal side of the vascular endothelial cells significantly reduced $\mathrm{CD}^{+} \mathrm{T}$ cell migration toward CCL21 in comparison with laminin $\alpha 4$ and PBS alone (Figure 8A). This effect was only observed when laminin $\alpha 5$ was present in excess of laminin $\alpha 4$. The presence of laminin $\alpha 4$, in amounts either equal to or in excess of laminin $\alpha 5$, did not affect Transwell migration and permitted migration despite the presence of laminin $\alpha 5$. Thus laminin $\alpha 4$ permitted $\mathrm{T}$ cell migration while laminin $\alpha 5$ inhibited migration across vascular endothelial cells; the effect of laminin $\alpha 4$ was dominant.

The path of $\mathrm{CD}^{+} \mathrm{T}$ cells during migration across the endothelial cell layer was observed with real-time microscopy, quantifying the track length (distance cells traveled) and velocity (speed of travel) over 30 minutes (Figure 8, $\mathrm{B}$ and $\mathrm{C}$ ). The track length and velocity were greatly reduced as the $\mathrm{T}$ cells migrated across endothelial cells overlaying laminin $\alpha 5$-coated inserts. The track length and velocity of $\mathrm{CD}^{+}$ $T$ cells migrating across endothelial cells were not as significantly reduced in the presence of laminin $\alpha 4$ alone or in combination with laminin $\alpha 5$. These data again showed that $\mathrm{T}$ cell movement over endothelial cells was hindered by laminin $\alpha 5$ but not $\alpha 4$. Further, the ratio of laminin $\alpha 4$ to $\alpha 5$ was important, as laminin $\alpha 4$ promoted $\mathrm{CD}^{+} \mathrm{T}$ cell migration across and through endothelial cells, dominantly counteracting the inhibitory effects of laminin $\alpha 5$.

To approximate the interactions of blood vascular endothelial cells, laminins, and $\mathrm{T}$ cells in vivo, $\mathrm{CD}^{+} \mathrm{T}$ cell adherence to and migration through endothelial cells in a laminar flow apparatus were examined. In this model, shear stress was applied by laminar flow to channels lined with endothelial cells over laminin coating treated with CCL21. Rolling and cell adherence were apparent almost immediately after addition of the $\mathrm{CD}^{+} \mathrm{T}$ cells to the channels. Five minutes after the addition of $\mathrm{CD}^{+} \mathrm{T}$ cells, increased numbers of $\mathrm{T}$ cells were adherent to the channels coated with laminin $\alpha 4$ only or in combination with laminin $\alpha 5$, as compared with channels coated with laminin $\alpha 5$ only (Figure 8D). As expected, there were significantly more adherent $\mathrm{T}$ cells in the presence of CCL21 as compared with no chemokine, showing that movements were chemokine dependent. Transmigration was greatly increased with laminin $\alpha 4$ as compared with laminin $\alpha 5$ (Figure $8 \mathrm{E}$ ). The percentage of adherent cells that had or had not migrated over time was also examined. $\mathrm{CD}^{+} \mathrm{T}$ cells migrated earlier through 
A

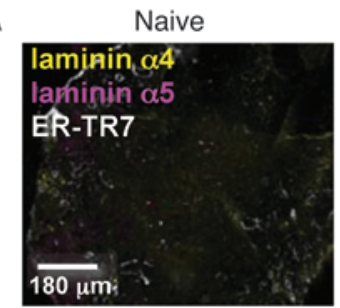

B

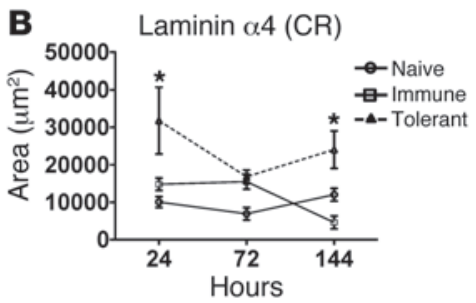

C

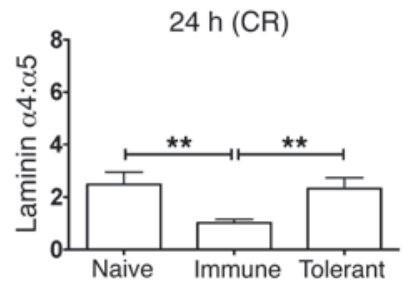

D Laminin $\alpha 4,24 \mathrm{~h}$ (HEV)

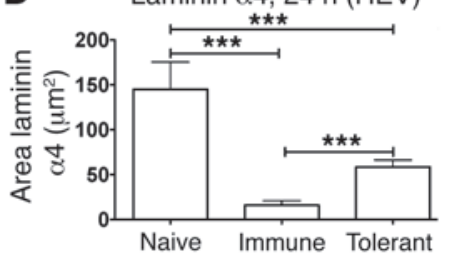

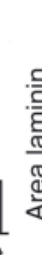

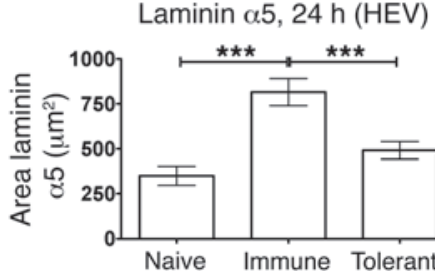

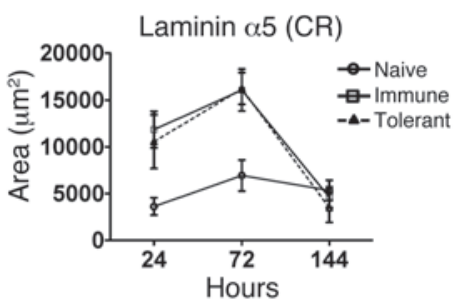

$72 \mathrm{~h}(\mathrm{CR})$
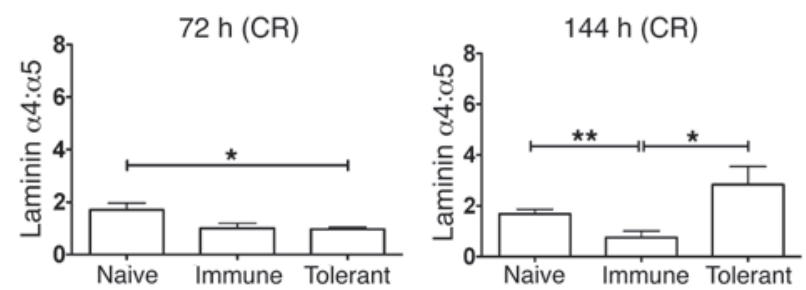

Ratio, $24 \mathrm{~h}$ (HEV)

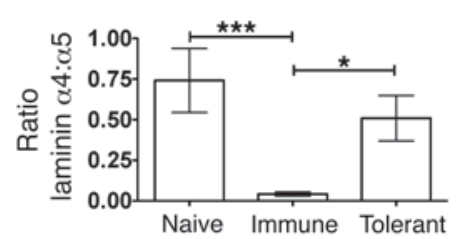

\section{Figure 7}

Laminin $\alpha 5$ and laminin $\alpha 4$ are differentially induced following the induction of immunity versus tolerance. LNs from naive, immune, and tolerant groups harvested 1-6 days after treatment and stained for laminin $\alpha 4$, laminin $\alpha 5$, and ER-TR7. (A) Representative images (original magnification, $\times 100$ ). (B) Total amount of staining for laminin $\alpha 4$ and laminin $\alpha 5$ in the cortical ridge (CR) quantified. (C) Ratio of laminin $\alpha 4$ to laminin $\alpha 5$ staining within the cortical ridge $(\times 100)$. (D) Total amounts of laminin $\alpha 4$ and laminin $\alpha 5$, and the ratio of laminin $\alpha 4$ to $\alpha 5$ surrounding the HEV $(\times 1,000)$. Data presented as mean \pm SEM. $(\mathbf{A}-\mathbf{C})$ $n=3-5$ mice per treatment; $1-4$ LNs per mouse $(\times 100)$. (D) $n=3$ mice per treatment; $1-4$ LNs per mouse; 30-100 HEVs per LN $(\times 1,000) .{ }^{*} P<0.05,{ }^{*} P<0.005$, ${ }^{* \star *} P<0.0005$. laminin $\alpha 4$-coated channels, and under this condition almost all adherent cells had gone through the endothelial cell layer at the latest time point examined (Supplemental Figure 3). Transmigration was significantly reduced in the channels coated with laminin $\alpha 5$ alone. Taken together, these data showed that the presence and ratio of laminin $\alpha 4$ and laminin $\alpha 5$ underlying the endothelial cells regulated how $\mathrm{T}$ cells adhered to and migrated across and through endothelial cells, under both static and shear conditions. Laminin $\alpha 4$ was more permissive for movement on and into the endothelial cell layer, while laminin $\alpha 5$ prevented such movements, and laminin $\alpha 4$ was dominant in its effects. These data are particularly notable in relation to the in vivo data for tolerance, where increased laminin $\alpha 4$ and $\alpha 4: \alpha 5$ ratios were coincident with increased movement of antigen-specific $\mathrm{CD} 4^{+} \mathrm{T}$ cells, Tregs, and pDCs through the HEV and into the CR.

Targeting the LN stromal fiber laminin $\alpha 5$ interferes with the induction of allograft rejection. The induction of tolerance was associated with LN remodeling and an increased ratio of laminin $\alpha 4$ to laminin $\alpha 5$, while immunity was associated with a decreased ratio of laminin $\alpha 4$ to laminin $\alpha 5$. We hypothesized that blocking the integrin-binding site of laminin $\alpha 5$ would interfere with immunity. Mice were injected in the footpad with $1 \mu \mathrm{g}$ anti-laminin $\alpha 5$ in conjunction with i.v. administration of DST and adoptive transfer of TEa T cells. Preliminary experiments demonstrated that this quantity of anti-laminin Ab was rapidly transported to the draining LN and bound the laminin $\alpha 5$ without being distributed systemically (not shown). Importantly, preliminary experiments revealed that larger i.v. doses of $\mathrm{Ab}$ and smaller footpad doses of $\mathrm{Ab}$ yielded the same results in terms of cell trafficking and LN remodeling (data not shown). Neither systemic nor footpad administration of anti-laminin Ab resulted in detectable toxicity. Mice appeared healthy, and all observed organs were of normal size and appearance. T cell, B cell, and DC distribution patterns within the spleen were normal (data not shown). As compared with antigen-specific $\mathrm{T}$ cell migration in immune HEVs, the distribution of cells within the HEVs of anti-laminin $\alpha 5$-treated LNs appeared more similar to the pattern observed in tolerant LNs (Figure 9A). In particular, the cells migrated more readily beyond the HEV and the surrounding basement membrane ER-TR7 ${ }^{+}$fibers into the CR. These data were commensurate with the in vitro migration results whereby laminin $\alpha 5$ impeded, while laminin $\alpha 4$ permitted, migration through the endothelium and associated basement membrane.

The accumulation of tolerance-inducing Tregs (Figure 9B), total $\mathrm{YAe}^{+}$cells (Figure 9C), and YAe ${ }^{+} \mathrm{PDCA}-1^{+}$pDCs (Figure 9D) was assessed in the CR of anti-laminin $\alpha 5$-treated mice. All of these cell types accumulated in the CR to a greater degree than in immune mice, and the percentages of accumulated cells were similar to the percentages observed in tolerant LNs. Hence, blocking the integrinbinding site of laminin $\alpha 5$ resulted in an accumulation of both 
A
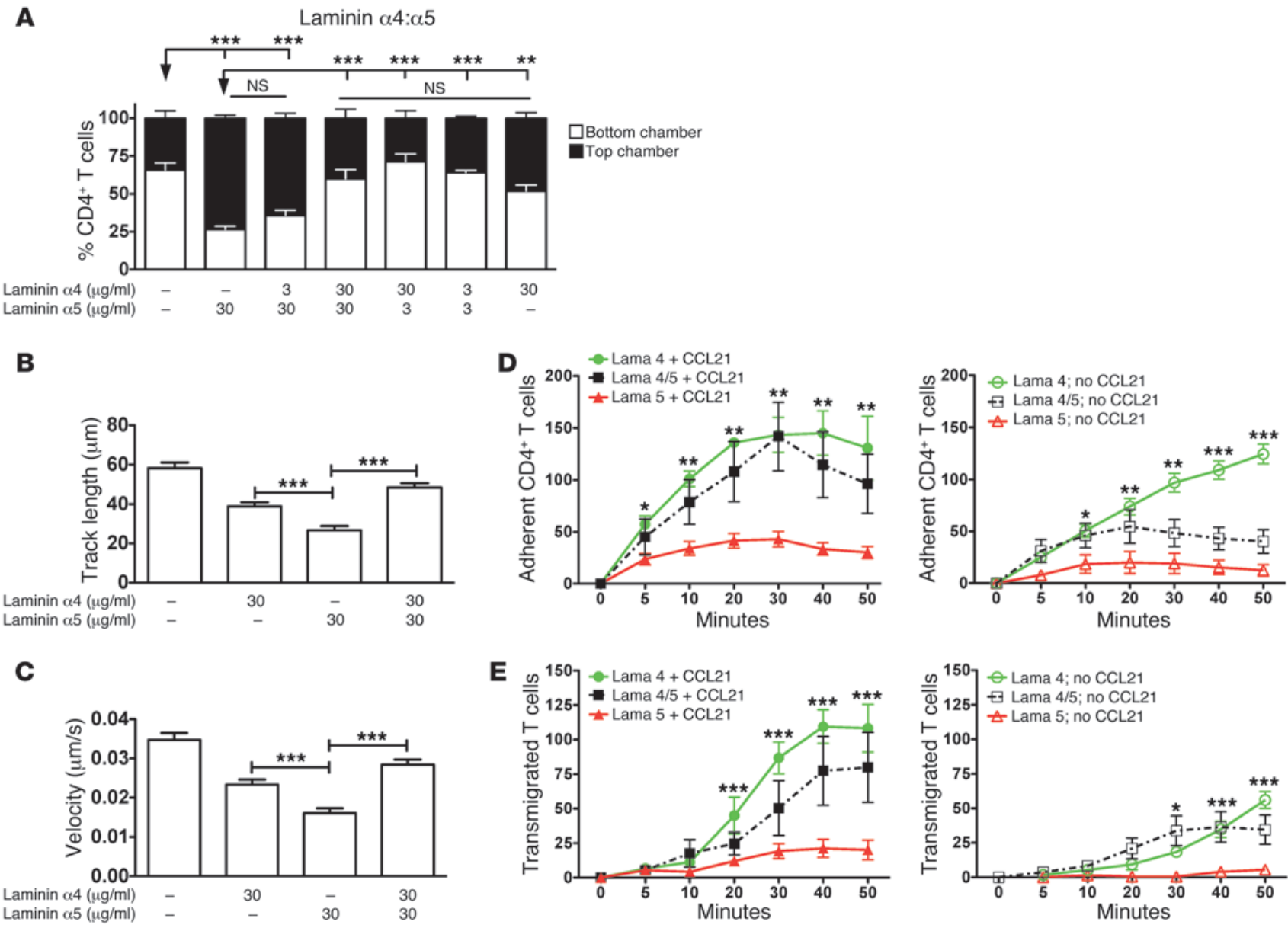

\section{Figure 8}

Laminins $\alpha 4$ and $\alpha 5$ differentially modulate CD4+ T cell motility and transendothelial migration. Transwell membrane inserts (A-C) or laminar flow channels ( $\mathbf{D}$ and $\mathbf{E}$ ) were coated with indicated concentrations of laminin $\alpha 4$ and/or $\alpha 5$ overnight (A-C) or for 1 hour (D and $\mathbf{E})$. MS-1 cells were added and grown to confluence in each apparatus. $0.5 \mu \mathrm{g}$ CCL21 was added to the bottom chambers $(\mathbf{A}-\mathbf{C})$ or passed through laminar flow channels ( $\mathbf{D}$ and $\mathbf{E})$ immediately before each migration assay. (A) Percentage of CD4+ cells located in the top chamber (black bars) and bottom chamber (white bars) 4 hours after their addition to upper Transwell chambers. (B and C) Track length (B) and velocity (C) of CD4+ T cell migration over MS-1 cells acquired at 1-minute intervals for 30 minutes after addition of CD4+ $T$ cells to the top Transwell chamber. (D) Adherence of CD4+ T cells to endothelial cells with (left panel) or without (right panel) CCL21 in laminar flow channels, imaged up to 50 minutes. (E) Transmigration of CD4 ${ }^{+} \mathrm{T}$ cells across endothelial cells in laminar flow channels with (left panel) and without (right panel) CCL21. Data presented as mean \pm SEM. $n=4$ replicates per experiment for 3 experiments. ${ }^{*} P<0.05,{ }^{* *} P<0.005,{ }^{* *} P<0.0005$. Lama $4 / 5$, Iaminin $\alpha 4 / \alpha 5$.

Tregs and APCs in the CR, and in cell migration and localization that were highly similar to those observed in tolerance.

As the ratio of laminin $\alpha 5$ to laminin $\alpha 4$ was reduced in tolerant versus immune recipients, we hypothesized that blocking the integrin-binding activities of laminin $\alpha 5$ would have a similar impact and favor the generation of immune suppression and tolerance. To test this hypothesis, otherwise untreated allograft recipients received $100 \mu \mathrm{g}$ anti-laminin $\alpha 5 \mathrm{Ab}$ i.v. on the day of transplantation. Graft function was monitored via abdominal palpation until function ceased. The addition of anti-laminin $\alpha 5$ resulted in a small but significant prolongation of graft survival (median survival time, 11.5 days vs. 8 days for unmodified recipients, $P=0.001$; Figure 9E). These data suggested that laminin $\alpha 5$-integrin interactions affected immunity and that inhibiting this interaction impaired immunity.

Targeting the LN stromal fiber laminin $\alpha 4$ interferes with the induction of allograft tolerance. We further hypothesized that interfering with laminin $\alpha 4$ function or decreasing the ratio of laminin $\alpha 4$ with respect to $\alpha 5$ would interfere with the establishment of tolerance. To test this hypothesis, mice were treated with a matrix metalloproteinase inhibitor (MMPi; $200 \mu \mathrm{g}$ i.v.) that targeted MMP-14, responsible for the degradation of laminin $\alpha 5$, along with DST plus anti-CD40L (tolerant + MMPi). Fluorescent immunohistochemistry revealed an increased amount of laminin $\alpha 5$ associated with the HEVs of tolerant + MMPi LNs (Figure 10A). Alternatively, the integrin-binding activities of laminin $\alpha 4$ were blocked with $1 \mu \mathrm{g}$ anti-laminin $\alpha 4$ injected into the footpad of tolerogen-treated mice.

MMPi and anti-laminin $\alpha 4$ both prevented tolerant-type migration of T cells into the HEVs (Figure 10B). Indeed, the patterns of antigen-specific T cell migration within the HEVs of tolerant + MMPi and tolerant + anti-laminin $\alpha 4$ mice were similar to the pattern of antigen-specific cell migration observed in immune HEVs. 

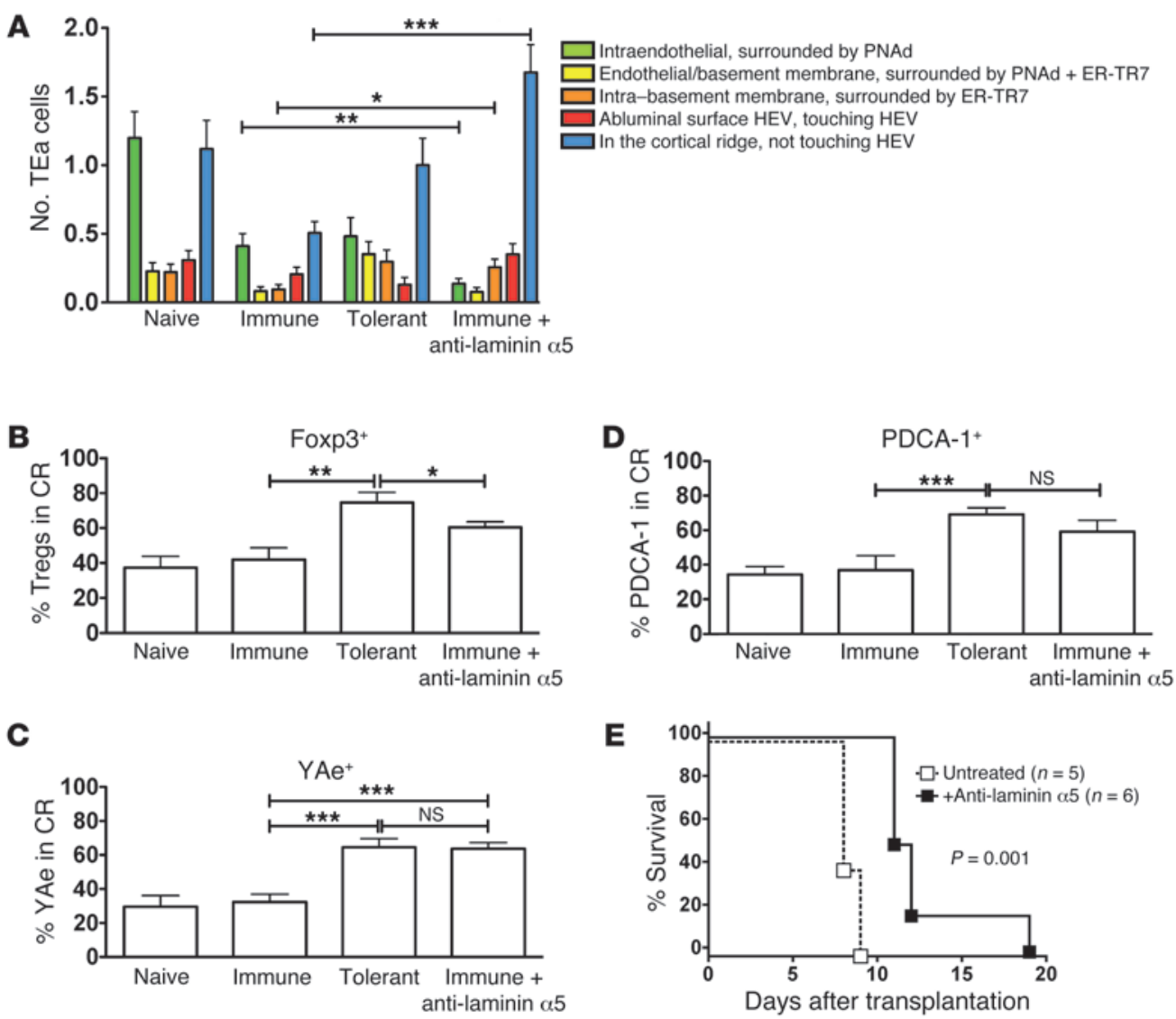

\section{Figure 9}

Targeting LN stromal fiber laminin $\alpha 5$ interferes with the induction of immunity and rejection. (A-D) Mice received DST + anti-CD40L (tolerant), DST only (immune), or DST + anti-laminin $\alpha 5 \mathrm{Ab}$ (1 $\mu \mathrm{g}$ in the footpad). Draining popliteal, inguinal, and para-aortic LN cryosections stained for the indicated markers. (A) CFSE ${ }^{+}$ $\mathrm{CD}^{+}$TEa cells were transferred and their distribution quantified after 4 hours. Figure 3B control data are presented for statistical comparison. (B-D) Mice were euthanized 24 hours after treatment, and the percentage of Foxp3 ${ }^{+}(\mathbf{B}), \mathrm{YAe}^{+}(\mathbf{C})$, and PDCA-1+ (D) cells in the cortical ridge was determined. (E) C57BL/6 mice received BALB/c cardiac allografts on day 0 and were left untreated or received $100 \mu \mathrm{g}$ anti-laminin $\alpha 5 \mathrm{Ab}$ i.v. Graft function was monitored and survival recorded (median survival time untreated $=8$ days, median survival time + anti-laminin $\alpha 5=11.5$ days). Data presented as mean \pm SEM $(\mathbf{A}-\mathbf{D})$ or percent survival (E). (A-D) $n=3-4$ mice per group; (E) $n=5-6$ mice per group. ${ }^{\star} P<0.05,{ }^{\star \star} P<0.005$, ${ }^{\star \star \star} P<0.0005$.

These data suggested that targeting the ratio of laminin $\alpha 4$ to laminin $\alpha 5$ or the integrin-binding function of laminin $\alpha 4$ altered the migration and location of antigen-specific cells within the HEVs.

Similarly, the migration of antigen-specific TEa T cells, Tregs, total $\mathrm{YAe}^{+}$cells, and $\mathrm{YAe}^{+} \mathrm{PDCA}-1^{+} \mathrm{pDC}$ in the $\mathrm{CR}$ was reduced by anti-laminin $\alpha 4 \mathrm{Ab}$ or MMPi (Figure 10, C-F). Hence, inhibition of laminin $\alpha 4$ integrin-binding function or its relative expression with respect to laminin $\alpha 5$ during tolerance induction altered migration of the relevant $\mathrm{T}$ cells and $\mathrm{pDCs}$ away from the CR. These data again were commensurate with the in vitro migration results whereby laminin $\alpha 5$ impeded while $\alpha 4$ permitted migration through the endothelium and associated basement membrane.

The impact of changing the laminin $\alpha 4: \alpha 5$ ratio or laminin $\alpha 4$ function on transplant survival was assessed. Tolerogen-treated mice were treated with $200 \mu \mathrm{g}$ MMPi on days -7 and 0 relative to transplantation, or with $100 \mu \mathrm{g}$ anti-laminin $\alpha 4 \mathrm{Ab}$ i.v. on days -7 and 0 relative to transplantation. Grafts were monitored for function until 40 days after transplantation (Figure 10G). Grafts placed in tolerant + MMPi mice had abnormal histology, with increased mononuclear cell infiltration and myocyte death (Figure 10H). Sixty percent of the anti-laminin $\alpha 4$ recipients rejected their grafts before day 40, while the remaining grafts had extensive mononuclear cell infiltrates and vascular edema (Figure $10 \mathrm{H})$. These data suggest that interfering with laminin $\alpha 4$ function or the ratio of laminin $\alpha 4$ to laminin $\alpha 5$ prevented tolerization.

\section{Discussion}

LNs and HEVs undergo structural and functional changes in response to antigenic challenge. LN hypertrophy and the concurrent increase in cellularity allow for increased opportunities for lymphocytes to encounter cognate antigen presented by APCs (53). To support the increase in LN size and influx of cells, the size of the arteriole that feeds the LN and the number and density of HEVs increase $(54,55)$. Angiogenesis is supported by BEC proliferation $(26,56)$ and promoted by DC and FRC interactions, resulting in FRC proliferation and VEGF production $(56,57)$. Subsequently, HEV structural modulation affects the types of cells permitted into the LN (58-60) and the subsequent immune response. HEVs upregulate E-selectin, CXCL12, CCL21, and CXCL9 in response to inflammation to attract both lymphocytes and pDCs (61). Activated HEV endothelial cells also express CD73, further controlling the migration of lymphocytes into the LN (62). Once lymphocytes establish firm interactions with the BECs, HEVs form pocket-like structures in which lymphocytes are maintained within the endothelium until sufficient space is available within the LN (18). Thus, through structural and functional changes, HEVs support LN remodeling to dictate the number and types of cell interactions that occur within the LN.

Our work demonstrated that alloantigen-reactive $\mathrm{CD}^{+} \mathrm{T}$ cells traffic uniquely through tolerant versus immune HEVs in an antigen-dependent manner. This observed acute antigen specificity was unexpected. In allografts, DCs reach processes through the vascular bed, present antigen, and promote $T$ cell transmigration (63). While this finding has not been confirmed in HEVs, it may contribute to the antigen specificity of differences in $\mathrm{T}$ cell trafficking. We also observed that $\mathrm{T}$ cells had extended interaction with HEVs following 
A
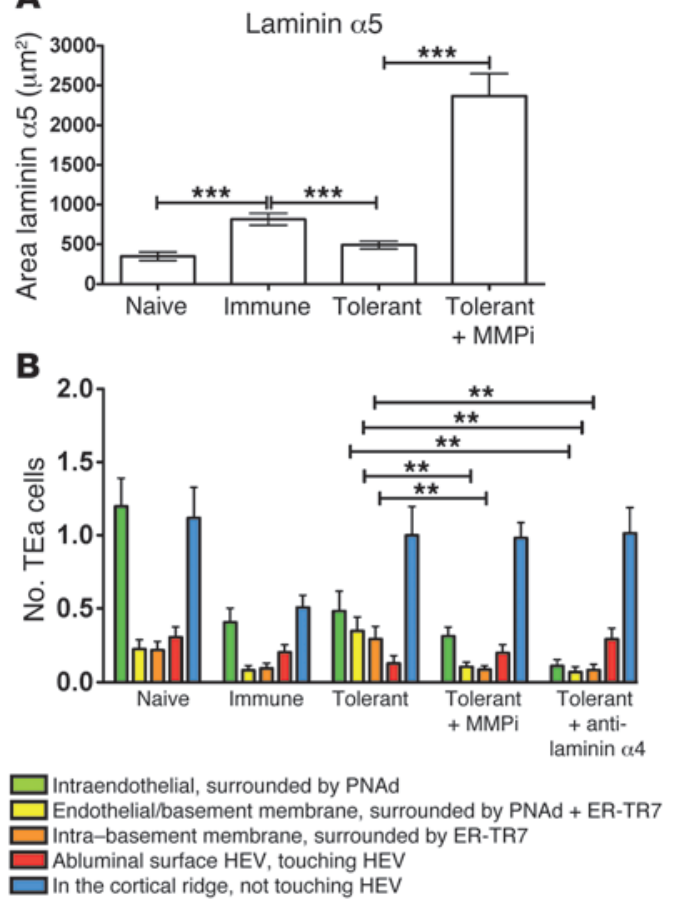

C

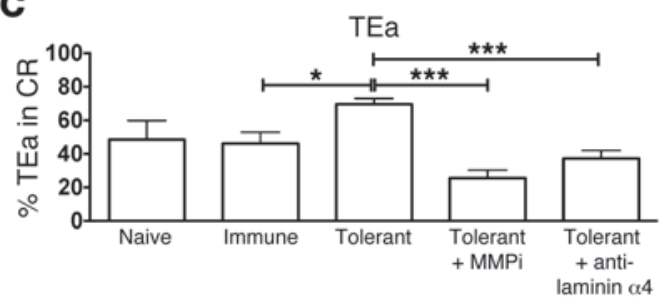

D
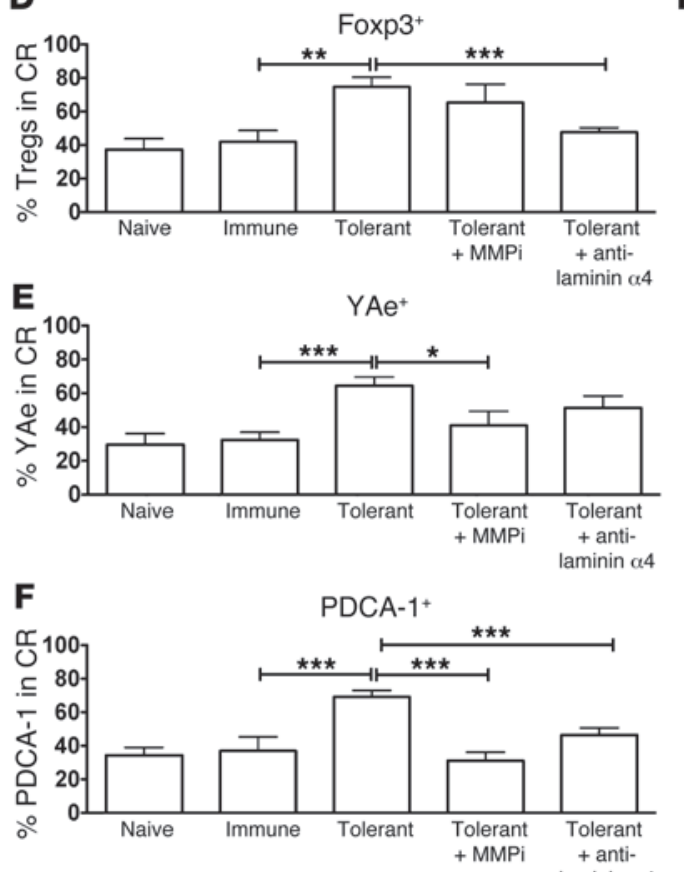

G

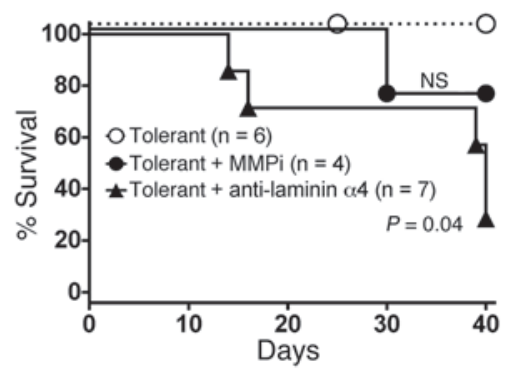

H

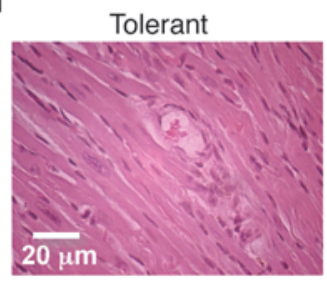

Tolerant + MMPi

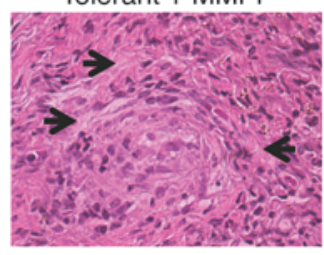

Tolerant + anti-laminin $\alpha 4$
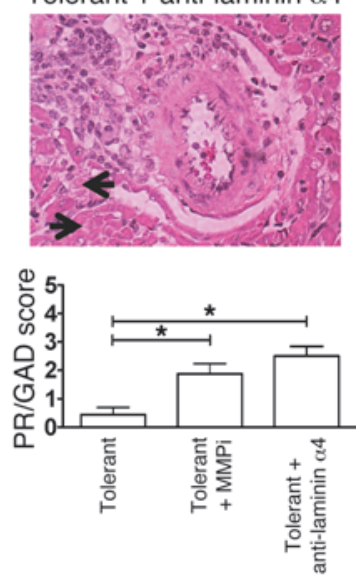

\section{Figure 10}

Targeting the LN stromal fiber laminin $\alpha 4$ interferes with tolerance induction. (A-F) Mice received DST + anti-CD40L (tolerant), DST only (immune), DST + anti-CD40L + MMPi (200 $\mu$ g, i.v. [tolerant + MMPi]), or DST + anti-CD40L + anti-laminin $\alpha 4$ Ab (1 $\mu \mathrm{g}$ in the footpad [tolerant + anti-laminin $\alpha 4]$ ). Draining popliteal, inguinal, and para-aortic LN cryosections stained for indicated markers. (A) Total amount of laminin $\alpha 5$ associated with HEVs quantified. Controls are repeated from Figure 7D for statistical comparison $(\times 1,000)$. (B) $\mathrm{CFSE}^{+} \mathrm{CD}^{+} \mathrm{TEa}^{+}$cells were transferred and their distribution quantified after 4 hours. Figure 3B control data are presented for statistical comparison. (C-F) Mice were euthanized 24 hours after treatment, and the percentage of CFSE ${ }^{+} \mathrm{TEa}^{+} \mathrm{CD} 4^{+} \mathrm{T}$ cells $(\mathbf{C})$ and Foxp3 ${ }^{+}(\mathbf{D}), \mathrm{YAe}^{+}(\mathbf{E})$, and PDCA $-1^{+}(\mathbf{F})$ cells in the cortical ridge was determined. Data from Figure 6, B-D, for naive, immune, and tolerant mice are included for statistical comparison. (G and H) C57BL/6 mice received DST + anti-CD40L, DST + anti-CD40L + MMPi (200 $\mu$ g, i.v., days -7, 0), or DST + anti-laminin $\alpha 4$ Ab (100 $\mu$ g i.v., days -7, 0). Recipients received $B A L B / c$ heterotopic cardiac allografts on day 0 . Graft function was monitored and survival recorded, and all mice were euthanized by day $40(\mathbf{G})$. Grafts were analyzed by H\&E staining (original magnification, $\times 600 ; \mathbf{H}$ ) and the parenchymal rejection/graft arterial disease (PR/GAD) score determined. Black arrows indicate areas of myocyte necrosis. Data presented as mean $\pm \mathrm{SEM}(\mathbf{A}-\mathbf{F}$ and $\mathbf{H})$ or percent survival $(\mathbf{G}) \cdot n=3-7$ mice per group. ${ }^{*} P<0.05,{ }^{* *} P<0.005,{ }^{* * *} P<0.0005$.

antigenic challenge, more so in tolerant than in immune LNs. In tolerant LNs, this prolonged contact manifested in an increased percentage of alloreactive cells adherent to the HEV, both within the lumen and in contact with the basement membrane. This interaction with the luminal side of the HEV may affect $T$ cell fate, since endothelial cells lining the HEV express FasL, suggesting they have the potential to affect $T$ cell survival at very early stages in T cell LN entry (64). The fate of $\mathrm{T}$ cells may also be affected by their remaining closely associated with the HEV following extravasation. Once lymphocytes exit the HEV, they are almost immediately exposed to the tolerance-inducing pDCs, alloantigen, and Tregs that home to and encircle HEVs following the induction of allograft tolerance
(3). In these ways, very early interactions between alloreactive T cells and the HEV may have long-reaching impact on T cell fate and the subsequent immune response.

The correct structure of the LN is important for the generation of appropriate immune responses. For example, following HIV infection, Tregs induce the production and deposition of collagen in the LN CR. This collagen effectively walls off the T cells entering the LN from the T cell zone, depriving the T cells of the IL-7 survival signal while depriving T-cell-zone FRCs of lymphocyte contact that is necessary for their survival. T cells and FRCs are both depleted, and the virus flourishes (19). Lymphocytic choriomeningitis virus targets FRCs, destroying the LN structure and inducing a state of 
immunodeficiency in the infected host (65). Restoration of the LN structure is necessary for regaining immunocompetence (66). Chronic inflammation in tumor-draining LNs results in a disruption of the stromal network and loss of T-cell-zone FRCs, leading to a decrease in CCL21 and a reduced T cell influx to the LNs (67). Our work demonstrated that $\mathrm{LN}$ remodeling also occurred acutely following tolerance induction. CR fibers became denser and were seeded with a combination of Tregs, pDCs presenting alloantigen, and antigen-specific TEa cells. One theory suggests that a dense FRC network, while perhaps not increasing the chances of naive $\mathrm{T}$ cells encountering their APC, does slow down $\mathrm{T}$ cell migration within and through the CR (68). Our findings couple a dense FRC network with an increased density of tolerance-inducing alloantigen-presenting $\mathrm{pDCs}$ and Tregs, suggesting that, following the induction of tolerance, not only do naive $\mathrm{T}$ cells move more slowly through the CR, but they also have an increased chance of encountering antigen and Tregs. Inhibition of fiber remodeling resulted in the abrogation of allograft tolerance, further demonstrating the importance of these structural changes. Interestingly, these and other structural changes were less pronounced, yet still detectable, 6-7 days after alloantigen exposure (data not shown), further demonstrating the plasticity of LN structure. Taken together, our data demonstrate that $\mathrm{LN}$ remodeling is an integral step in the generation of transplant tolerance, creating a microenvironment suitable for tolerogenic interactions among DCs and T cells.

The HEV basement membranes and the structural fibers of the CR contain both laminin $\alpha 4$ and $\alpha 5$ (69). These proteins have highly conserved functions and can serve as anchors for endothelial cells under shear stress conditions (70). Laminin $\alpha 4$ is permissive for transmigration of neutrophils, $T$ cells, and even parasites through blood vessels, whereas laminin $\alpha 5$ is inhibitory (71-73). Laminins have a variety of structural, adhesive, and stimulatory functions, and their importance is underlined by the findings that laminin $\alpha 4$-null mice experience extensive hemorrhage and impaired microvascular growth (32), and that laminin $\alpha 5$ deficiency results in embryonic lethality (52). The G domains of both laminin $\alpha 4$ and $\alpha 5$ serve as ligands for $\mathrm{T}$ cell integrins, promoting and affecting $\mathrm{T}$ cell trafficking both as T cells leave the HEV and within the LN parenchyma (74-76). We observed laminin $\alpha 5$ but not laminin $\alpha 4$ to have a modest impact on $\mathrm{T}$ cell proliferation and activation, and negligible impact on Treg differentiation, following coculture with anti-CD3, IL-2, and APC stimulation (data not shown). In contrast, we observed major differences in $\mathrm{T}$ cell migration across laminin proteins, even in the absence of endothelial cells (Figure 8 and data not shown). Laminin $\alpha 4$ and laminin $\alpha 5$ were differentially remodeled following the induction of tolerance versus immunity and heavily influenced $\mathrm{T}$ cell migration along, and transmigration through, HEVs. Inhibition of $\mathrm{T}$ cell binding to laminin $\alpha 5$ was sufficient to alter $\mathrm{T}$ cell migratory patterns and prolong graft function in rejecting allograft recipients. Leukocytes preferentially exit blood vessels at points in the basement membrane where laminin $\alpha 5$ expression is low $(77,78)$. By blocking laminin $\alpha 5$ we may have artificially created laminin $\alpha 5-$ low regions in the HEVs, allowing for the transmigration of alloreactive $T$ cells and the accumulation of $\mathrm{pDCs}$ and Tregs within the CR. These findings suggest remodeling of laminin $\alpha 5$ as a molecular control driving the generation of immunity versus tolerance.

Increased laminin $\alpha 4$ was observed following the induction of tolerance and was permissive for the transmigration of $\mathrm{T}$ cells. Both blockade of laminin $\alpha 4$ and overexpression of laminin $\alpha 5$ were sufficient to alter $\mathrm{T}$ cell migratory patterns and induce graft inflammation in tolerant allograft recipients. Blockade of laminin $\alpha 4$ could potentially affect 2 steps of tolerance induction, $\mathrm{LN}$ restructuring and $\mathrm{T}$ cell trafficking. As discussed above, HEV angiogenesis occurs concurrently with LN hypertrophy (54). Treating tolerant mice with anti-laminin $\alpha 4$ may inhibit this restructuring, as endothelial cell interactions with the $G$ domain of laminin $\alpha 4$ are necessary for angiogenesis $(79,80)$. Blockade of laminin $\alpha 4$ and overexpression of laminin $\alpha 5$ also likely affected the ability of T cells to exit the HEVs and traffic through the CR, as we observed less association of alloreactive $\mathrm{T}$ cells and tolerance-inducing $\mathrm{PDCs}$ and Tregs encircling the HEVs and within the CR. These changes in cell positioning potentially reduced the necessary interactions to induce allograft tolerance. These results suggest laminin $\alpha 4$ remodeling as a necessary step in tolerance induction.

Our study demonstrates specific remodeling of the HEVs and LNs correlated with allograft tolerance or with rejection. Laminin $\alpha 4$ and $\alpha 5$ were differentially regulated following the induction of tolerance versus immunity, and these differences were associated with differential kinetics of alloreactive $T$ cell trafficking within and extravasation through HEVs. These differences in laminin expression were also reflected by the localization of alloantigen-reactive $\mathrm{T}$ cells within the $\mathrm{CR}$, and the colocalization of alloantigen-reactive $\mathrm{T}$ cells, alloantigen, $\mathrm{pDCs}$, and Tregs following the induction of tolerance but not immunity. As intervention in these structural modulations resulted in lasting impacts on graft survival, laminin proteins represent a target for acutely generating transplant tolerance or enhancing immunogenicity of antigens.

\section{Methods}

Mice. C57BL/6 (H-2 $)$, BALB/c (H-2 $)$, and OT-II-Tg (B6.Cg-Tg (TcraTcrb) $425 \mathrm{Cbn} / \mathrm{J})$ mice were purchased from Jackson Laboratory. $\mathrm{T}$ cell $\mathrm{Tg}$ mice expressing the TEa TCR (recognizes I- $\mathrm{A}^{\mathrm{e}}$ antigen in I- $\mathrm{A}^{\mathrm{b}}$ complex) were acquired from A.Y. Rudensky (Memorial Sloan Kettering Cancer Center, New York, NY; ref. 81). Mice were used at 8-12 weeks of age, and all experiments were performed with age- and sex-matched mice. Animals were housed under specific-pathogen-free conditions.

Tolerance induction. DST cells were obtained from BALB/c spleens that were homogenized and passed through a $70-\mu \mathrm{m}$ cell strainer and treated with ACK lysis buffer (Lonza). Seven days before cardiac allograft transplantation, mice were tolerized i.v. with $1 \times 10^{7} \mathrm{DST}$ cells and anti-CD40L $\mathrm{mAb}$ (clone MR1; $0.25 \mathrm{mg}$ i.v.; Bio X Cell). Anti-CD40L was administered again at day -4 , day 0 , and day +4 relative to transplantation. Immune mice were left untreated before cardiac allograft transplantation.

Cardiac allograft procedure and mouse treatments. C57BL/6 mice (10-12 weeks) received heterotopic cardiac allograft transplants from sex-matched donor BALB/c mice (6-8 weeks) as previously described (82). Tolerized mice were treated with anti-laminin $\alpha 4 \mathrm{Ab}(100 \mu \mathrm{g}$ i.v., days -7 and 0 ; Novus Biologicals) or MMP-14 inhibitor (MMPi) (200 $\mu$ g i.v., days -7 and 0; GenScript). Immune mice were treated with anti-laminin $\alpha 5 \mathrm{Ab}(100 \mu \mathrm{g}$ i.v., day 0; Novus Biologicals) after surgery (Supplemental Table 1, in vivo). Graft function was monitored daily by abdominal palpitation. At either time of rejection or 40 days after transplantation, mice were euthanized and the donor heart was excised, fixed with $10 \%$ paraformaldehyde, and embedded in paraffin. Graft parenchymal and perivascular inflammation and cellular infiltration were determined using H\&E staining. Masson's trichrome staining was used to determine the degree of fibrosis in graft tissue sections. Parenchymal rejection and graft arterial disease (PR/GAD) scoring was based on a modified protocol published by the International Society for Heart and Lung Transplantation (83-85). 
Cell preparations and T cell adoptive transfer. LNs and spleen were collected from TEa Tg or OT-II Tg mice, and tissue was passed through a $70-\mu \mathrm{m}$ cell strainer for homogenization. $\mathrm{CD} 4^{+} \mathrm{T}$ cell isolation was completed using the EasySep Mouse $\mathrm{CD}^{+} \mathrm{T}$ cell Enrichment Kit according to the manufacturer's recommendations (StemCell Technologies). Purity of 95\%-99\% was confirmed by flow cytometry for each experiment. Cells were labeled with CFSE or Qtracker 655 according to the manufacturer's instructions (Thermo Fisher Scientific Inc.). Mice were given $20 \times 10^{6}$ cells i.v. at the time of tolerance or immune induction. As indicated, tolerized mice were treated with anti-lami$\operatorname{nin} \alpha 4 \mathrm{mAb}$ (1 $\mu \mathrm{g}$ via footpad injection, day -8$)$ or MMPi (100 $\mu$ g i.v., day 0). Anti-laminin- $\alpha 5 \mathrm{Ab}$ was administered $(1 \mu \mathrm{g}$ via footpad injection, day -8$)$.

Multiphoton intravital microscopy. Mice were anesthetized with isoflurane and abdominal fur removed with depilatory. Mice were immobilized to a restraint apparatus and maintained on a $37^{\circ} \mathrm{C}$ microscope stage. The abdominal cavity surrounding the right inguinal $\mathrm{LN}$ was opened and fixed to the restraint apparatus. The $\mathrm{LN}$ was exposed, with careful attention to avoid severing blood and lymphatic vessels. Mice received a single injection containing $20 \times 10^{6} \mathrm{CFSE}^{+} \mathrm{CD}^{+} \mathrm{TEa}$ TCR Tg T cells, $20 \times 10^{6}$ Qtracker $655^{+} \mathrm{CD} 4^{+}$OT-II TCR Tg T cells, $10^{7}$ DST cells, and $2 \mathrm{mg}$ TRITC-conjugated 70-kDa dextran (Thermo Fisher Scientific Inc.), with or without $0.25 \mathrm{mg}$ i.v. anti-CD40L mAb. Maintaining a liquid column of $1 \times$ PBS between the $\mathrm{LN}$ and submergible $\times 200$ optic, $\mathrm{LNs}$ were imaged from 20 to 240 minutes after cell transfer using a Zeiss 710 NLO OPO multiphoton microscope and ZEN 2009 software (Carl Zeiss). Images were analyzed using Volocity image analysis software (PerkinElmer). Circulating and rolling cells (Figure 1, C and D) were manually quantified in 200 frames per 800 -frame sequence and normalized for total events per sequence.

Immunohistochemistry. LNs, spleen, and heart were excised and immediately submerged in OCT compound (Sakura Finetek) or fixed using paraformaldehyde. Tissues in OCT were quickly frozen using dry ice, then kept at $-80^{\circ} \mathrm{C}$ for long-term storage. Whole organs fixed in paraformaldehyde were subsequently embedded in paraffin, sectioned, and stained using H\&E. LN cryosections were cut in triplicate at $5 \mu \mathrm{m}$ using a Microm HM 550 cryostat (Thermo Fisher Scientific Inc.). Sections were fixed with cold acetone for 5 minutes, then washed in PBS or left unfixed (Lonza) for fluorescent microscopy. Primary Ab's were diluted between 1:20 and 1:200 in PBS and incubated for 1 hour in a humidified chamber (Supplemental Table 2). Sections were washed 1 time with PBS, and secondary Ab was applied at a 1:501:400 dilution for 30 minutes (Supplemental Table 3). Slides were washed in PBS for 5 minutes, coverslipped, and imaged under a fluorescent microscope. Slides for confocal microscopy were fixed with $4 \%$ paraformaldehyde and treated with $1 \%$ glycerol in PBS before coverslipping and imaging. Images were analyzed with Volocity image analysis software (PerkinElmer).

Transmigration assay. Laminin $\alpha 4$ (R\&D Systems Inc.) and laminin-511 (containing the $\alpha 5$ chain; BioLamina) proteins were diluted in PBS and coated onto $5-\mu \mathrm{m}$ porous Transwell inserts set in a 24-well plate (Costar; Corning Inc.) at $4{ }^{\circ} \mathrm{C}$ overnight. MS- 1 cells (American Type Culture Collection) were removed from liquid nitrogen, suspended in media, and propagated onto a $75-\mathrm{cm}^{2}$ tissue culture flask for 2-3 days in 10\% DMEM. Two to three days before the transmigration assay, MS- 1 cells were added into the top of the laminin-coated Transwell insert. MS- 1 cells were added at a dose of 75,000 to 150,000 cells per insert and incubated at $37^{\circ} \mathrm{C}, 5 \% \mathrm{CO}_{2}$ for 1-2 days. To confirm MS-1 cell confluence, $\mathrm{H} \& \mathrm{E}$ staining was performed. $\mathrm{CD}^{+} \mathrm{T}$ cells were isolated from $\mathrm{C} 57 \mathrm{BL} / 6$ mice as described above. $\mathrm{CD}^{+} \mathrm{T}$ cell concentration was adjusted to $1 \times 10^{8}$ cells per milliliter, and cells were fluorescently labeled with CFSE $(2.5 \mu \mathrm{g} / \mathrm{ml})$ for 10 minutes. Six hundred microliters of fresh $0.5 \%$ BSA media plus $0.5 \mu \mathrm{g} / \mathrm{ml}$ of CCL 21 was added to the bottom well; $5 \times 10^{5}$ CFSE-labeled $\mathrm{CD} 4^{+} \mathrm{T}$ cells were added to the top of the insert, and the plate was incubated at $37^{\circ} \mathrm{C}$ in $5 \% \mathrm{CO}_{2}$ for 3 hours. Cells were quantified in the top and bottom wells of the chamber to assess migration across inserts.

Cellular perfusion assay. In vitro perfusion experiments were performed using a BioFlux 1000 system (Fluxion Biosciences) coupled with an automated Axio Observer Z1 microscope (Carl Zeiss). Perfusion and imaging sequences were controlled using system software from a standard desktop computer. Twenty-four-channel, 0- to 20-dyne, BioFlux 1000 plates were initially coated with laminin $\alpha 4$, laminin-511, or laminin $\alpha 4$ plus laminin-511 $(50 \mu \mathrm{g} / \mathrm{ml})$ for 1 hour at $37^{\circ} \mathrm{C}$ in $5 \% \mathrm{CO}_{2}$. Channels were washed once with HBSS plus $0.5 \%$ BSA and seeded with MS- 1 cells suspended in DMEM plus $10 \%$ FBS. The plate was then placed in a $37^{\circ} \mathrm{C}, 5 \% \mathrm{CO}_{2}$ incubator for 2 days or until MS-1 cells reached confluence, determined by visual examination of each channel. CCL21 $(0.5 \mu \mathrm{g} / \mathrm{ml})$ was added to selected channels for 6-12 hours before the addition of $\mathrm{CFSE}^{+} \mathrm{CD} 4^{+} \mathrm{T}$ cells. One hundred microliters of $\mathrm{CD} 4^{+} \mathrm{T}$ cells $\left(2.5 \times 10^{6}\right.$ to $\left.3.0 \times 10^{6} \mathrm{cells} / \mathrm{ml}\right)$ were perfused through channels $\left(0.5\right.$ dynes $\left./ \mathrm{cm}^{2}, 37^{\circ} \mathrm{C}\right)$. Bright-field and fluorescent images $(\times 200,10-\mathrm{ms}$ and 50-ms exposures, respectively) were obtained from 4 representative positions in each channel for a minimum of 30 minutes. Adherence and transmigration were determined using Volocity image analysis software (PerkinElmer).

Statistics. Data were analyzed with Prism 4.0c software (GraphPad Software Inc.) using Student's unpaired 2-tailed $t$ tests (for single variable differences), or 1- or 2-way ANOVA (for multiple variable differences) with Tukey's or Bonferroni post-tests, as appropriate. $P$ values of 0.05 or less were considered statistically significant.

Study approval. All mouse procedures were performed in accordance with protocols approved by the Institutional Animal Care and Utilization Committee at the University of Maryland School of Medicine.

\section{Acknowledgments}

This work was supported by grants R01-AI062765 and R56AI72039 (to J.S. Bromberg) and T32-AI078892 and HL007698 (to B.E. Burrell) from the NIH.

Received for publication October 10, 2013, and accepted in revised form January 23, 2014.

Address correspondence to: Jonathan S. Bromberg, Department of Surgery, University of Maryland School of Medicine, 29 S. Greene Street, Suite 200, Baltimore, Maryland 20201, USA. Phone: 410.328.0008; Fax: 410.328.6343; E-mail: jbromberg@ smail.umaryland.edu. Or to: Bryna E. Burrell, Immune Tolerance Network, 3 Bethesda Metro Center, Suite 400, Bethesda, Maryland 20814, USA. Phone: 240.235.6130; Fax: 240.235.6198; E-mail: bburrell@immunetolerance.org.

Bryna E. Burrell's present address is: Immune Tolerance Network, Bethesda, Maryland, USA.

Daiki Iwami's present address is: Hokkaido University Hospital, Department of Urology, Kita-ku, Sapporo, Hokkaido, Japan.

Kristi J. Warren's present address is: Johns Hopkins Medicine, Anesthesiology \& Critical Care Medicine, Baltimore, Maryland, USA.
1. Burrell BE, et al. Tolerance and lymphoid organ structure and function. Front Immunol. 2011;2:64.

2. Willard-Mack CL. Normal structure, function, and histology of lymph nodes. Toxicol Pathol.
2006:34(5):409-424

3. Ochando JC, et al. Alloantigen-presenting plasmacytoid dendritic cells mediate tolerance to vascularized grafts. Nat Immunol. 2006;7(6):652-662.
4. Burrell BE, Bromberg JS. Fates of $\mathrm{CD}^{+} \mathrm{T}$ cells in a tolerant environment depend on timing and place of antigen exposure. Am J Transplant. 2012; 12(3):576-589. 
5. Ochando JC, et al. Lymph node occupancy is required for the peripheral development of alloantigen-specific Foxp3+ regulatory T cells. J Immunol. 2005;174(11):6993-7005

6. Nakayama Y, Bromberg JS. Lymphotoxin-beta receptor blockade induces inflammation and fibrosis in tolerized cardiac allografts. Am J Transplant. 2012;12(9):2322-2334.

7. Bai Y, et al. L-selectin-dependent lymphoid occupancy is required to induce alloantigen-specific tolerance. J Immunol. 2002;168(4):1579-1589.

8. Zhang N, et al. Regulatory $\mathrm{T}$ cells sequentially migrate from inflamed tissues to draining lymph nodes to suppress the alloimmune response. Immunity. 2009;30(3):458-469.

9. Tang $\mathrm{Q}$, et al. Visualizing regulatory $\mathrm{T}$ cell control of autoimmune responses in nonobese diabetic mice. Nat Immunol. 2006;7(1):83-92

10. Gupta $S$, et al. Allograft rejection is restrained by short-lived TIM- $3^{+} \mathrm{PD}-1^{+} \mathrm{Foxp} 3^{+}$Tregs. J Clin Invest. 2012;122(7):2395-2404.

11. Golshayan D, Jiang S, Tsang J, Garin MI, Mottet C, Lechler RI. In vitro-expanded donor alloantigen-specific $\mathrm{CD} 4^{+} \mathrm{CD} 25^{+}$regulatory $\mathrm{T}$ cells promote experimental transplantation tolerance. Blood. 2007;109(2):827-835.

12. Manicassamy S, Pulendran B. Dendritic cell control of tolerogenic responses. Immunol Rev. 2011; 241(1):206-227.

13. Vitali C, et al. Migratory, and not lymphoid-resident, dendritic cells maintain peripheral self-tolerance and prevent autoimmunity via induction of iTreg cells. Blood. 2012;120(6):1237-1245.

14. Katakai T, Hara T, Lee JH, Gonda H, Sugai M, Shimizu A. A novel reticular stromal structure in lymph node cortex: an immuno-platform for interactions among dendritic cells, T cells and B cells. Int Immunol. 2004;16(8):1133-1142.

15. Shakhar G, et al. Stable T cell-dendritic cell interactions precede the development of both tolerance and immunity in vivo. Nat Immunol. 2005;6(7):707-714.

16. Girard JP, Moussion C, Forster R. HEVs, lymphatics and homeostatic immune cell trafficking in lymph nodes. Nat Rev Immunol. 2012;12(11):762-773.

17. Liu X, et al. Tolerance induction towards cardiac allografts under costimulation blockade is impaired in CCR7-deficient animals but can be restored by adoptive transfer of syngeneic plasmacytoid dendritic cells. Eur J Immunol. 2011;41(3):611-623.

18. Mionnet $\mathrm{C}$, et al. High endothelial venules as traffic control points maintaining lymphocyte population homeostasis in lymph nodes. Blood. 2011;118(23):6115-6122.

19. Zeng M, et al. Cumulative mechanisms of lymphoid tissue fibrosis and T cell depletion in HIV-1 and SIV infections. J Clin Invest. 2011;121(3):998-1008.

20. Bajenoff $M$, et al. Stromal cell networks regulate lymphocyte entry, migration, and territoriality in lymph nodes. Immunity. 2006;25(6):989-1001.

21. Zhu M, Fu YX. The role of core TNF/LIGHT family members in lymph node homeostasis and remodeling. Immunol Rev. 2011;244(1):75-84.

22. Kumar V, et al. Global lymphoid tissue remodeling during a viral infection is orchestrated by a $B$ cell-lymphotoxin-dependent pathway. Blood. 2010; 115(23):4725-4733

23. Onder L, et al. IL-7-producing stromal cells are critical for lymph node remodeling. Blood. 2012; 120(24):4675-4683.

24. Abe J, et al. B cells regulate antibody responses through the medullary remodeling of inflamed lymph nodes. Int Immunol. 2011;24(1):17-27.

25. Kumamoto Y, Mattei LM, Sellers S, Payne GW, Iwasaki A. CD4 ${ }^{+} \mathrm{T}$ cells support cytotoxic T lymphocyte priming by controlling lymph node input. Proc Natl Acad Sci U S A. 2011;108(21):8749-8754.

26. Liao S, Ruddle NH. Synchrony of high endothelial venules and lymphatic vessels revealed by immuni- zation. J Immunol. 2006;177(5):3369-3379.

27. Webster B, Ekland EH, Agle LM, Chyou S, Ruggieri $\mathrm{R}, \mathrm{Lu}$ TT. Regulation of lymph node vascular growth by dendritic cells. J Exp Med. 2006;203(8):1903-1913.

28. Katakai T, Hara T, Sugai M, Gonda H, Shimizu A. Lymph node fibroblastic reticular cells construct the stromal reticulum via contact with lymphocytes. J Exp Med. 2004;200(6):783-795.

29. Van Vliet E, Melis M, Foidart JM, Van Ewijk W. Reticular fibroblasts in peripheral lymphoid organs identified by a monoclonal antibody. J Histochem Cytochem. 1986;34(7):883-890.

30. Frieser M, et al. Cloning of the mouse laminin $\alpha 4$ cDNA. Expression in a subset of endothelium. Eur J Biochem. 1997;246(3):727-735.

31. Sorokin LM, Pausch F, Frieser M, Kroger S, Ohage E, Deutzmann R. Developmental regulation of the laminin $\alpha 5$ chain suggests a role in epithelial and endothelial cell maturation. Dev Biol. 1997; 189(2):285-300.

32. Thyboll J, et al. Deletion of the laminin $\alpha 4$ chain leads to impaired microvessel maturation. Mol Cell Biol. 2002;22(4):1194-1202.

33. Geberhiwot T, et al. Laminin-8 $(\alpha 4 \beta 1 \gamma 1)$ is synthesized by lymphoid cells, promotes lymphocyte migration and costimulates $\mathrm{T}$ cell proliferation. J Cell Sci. 2001;114(pt 2):423-433.

34. Pouliot N, Kusuma N. Laminin-511: a multi-functional adhesion protein regulating cell migration, tumor invasion and metastasis. Cell Adh Migr. 2013; 7(1):142-149.

35. Domogatskaya A, Rodin S, Boutaud A, Tryggvason K. Laminin- 511 but not $-332,-111$, or -411 enables mouse embryonic stem cell self-renewal in vitro. Stem Cells. 2008;26(11):2800-2809.

36. Wu C, et al. Endothelial basement membrane laminin $\alpha 5$ selectively inhibits T lymphocyte extravasation into the brain. Nat Med. 2009;15(5):519-527.

37. Song J, et al. Extracellular matrix of secondary lymphoid organs impacts on B-cell fate and survival. Proc Natl Acad Sci US A. 2013;110(31):E2915-E2924.

38. Parker DC, et al. Survival of mouse pancreatic islet allografts in recipients treated with allogeneic small lymphocytes and antibody to CD40 ligand. Proc Natl Acad Sci U S A. 1995;92(21):9560-9564.

39. Durie FH, et al. Antibody to the ligand of CD40, gp39, blocks the occurrence of the acute and chronic forms of graft-vs-host disease. J Clin Invest. 1994; 94(3):1333-1338

40. Markees TG, et al. Long-term survival of skin allografts induced by donor splenocytes and anti-CD 154 antibody in thymectomized mice requires CD4(+) $\mathrm{T}$ cells, interferon- $\gamma$, and CTLA4. $J$ Clin Invest. 1998;101(11):2446-2455.

41. Taylor PA, Noelle RJ, Blazar BR. CD4(+)CD25(+) immune regulatory cells are required for induction of tolerance to alloantigen via costimulatory blockade. J Exp Med. 2001;193(11):1311-1318.

42. Quezada SA, et al. Mechanisms of donor-specific transfusion tolerance: preemptive induction of clonal T-cell exhaustion via indirect presentation. Blood. 2003;102(5):1920-1926.

43. Zheng XX, et al. Blockade of CD40L/CD40 costimulatory pathway in a DST presensitization model of islet allograft leads to a state of Allo-Ag specific tolerance and permits subsequent engraftment of donor strain islet or heart allografts. Transplant Proc. 1999;31(1-2):627-628.

44. Iwakoshi NN, Mordes JP, Markees TG, Phillips NE, Rossini AA, Greiner DL. Treatment of allograft recipients with donor-specific transfusion and anti-CD154 antibody leads to deletion of alloreactive $\mathrm{CD}^{+} \mathrm{T}$ cells and prolonged graft survival in a CTLA4-dependent manner. J Immunol. 2000; 164(1):512-521.

45. Lu L, et al. Increased apoptosis of immunoreactive host cells and augmented donor leukocyte chimerism, not sustained inhibition of B7 molecule expression are associated with prolonged cardiac allograft survival in mice preconditioned with immature donor dendritic cells plus anti-CD40L mAb. Transplantation. 1999;68(6):747-757.

46. van Maurik A, Fazekas de St Groth B, Wood KJ, Jones ND. Dependency of direct pathway $\mathrm{CD}^{+} \mathrm{T}$ cells on CD40-CD154 costimulation is determined by nature and microenvironment of primary contact with alloantigen. JImmunol. 2004;172(4):2163-2170.

47. Sanchez-Fueyo A, Domenig C, Strom TB, Zheng $\mathrm{XX}$. The complement dependent cytotoxicity (CDC) immune effector mechanism contributes to anti-CD154 induced immunosuppression. Transplantation. 2002;74(6):898-900.

48. Monk NJ, et al. Fc-dependent depletion of activated $T$ cells occurs through CD40L-specific antibody rather than costimulation blockade. Nat Med. 2003;9(10):1275-1280.

49. Wood SC, Lu G, Burrell BE, Bishop DK. Transplant acceptance following anti-CD4 versus anti-CD40L therapy: evidence for differential maintenance of graft-reactive T cells. Am J Transplant. 2008; 8(10):2037-2048

50. Csencsits K, Burrell BE, Lu G, Eichwald EJ, Stahl GL, Bishop DK. The classical complement pathway in transplantation: unanticipated protective effects of $\mathrm{C} 1 \mathrm{q}$ and role in inductive antibody therapy. $\mathrm{AmJ}$ Transplant. 2008;8(8):1622-1630.

51. Itoh M, et al. Thymus and autoimmunity: production of $\mathrm{CD} 25^{+} \mathrm{CD} 4^{+}$naturally anergic and suppressive $\mathrm{T}$ cells as a key function of the thymus in maintaining immunologic self-tolerance. J Immunol. 1999;162(9):5317-5326.

52. Miner JH, Cunningham J, Sanes JR. Roles for laminin in embryogenesis: exencephaly, syndactyly, and placentopathy in mice lacking the laminin $\alpha 5$ chain. J Cell Biol. 1998;143(6):1713-1723.

53. Angeli $\mathrm{V}$, et al. B cell-driven lymphangiogenesis in inflamed lymph nodes enhances dendritic cell mobilization. Immunity. 2006;24(2):203-215.

54. Kumar V, Chyou S, Stein JV, Lu TT. Optical projection tomography reveals dynamics of HEV growth after immunization with protein plus CFA and features shared with HEVs in acute autoinflammatory lymphadenopathy. Front Immunol. 2012;3:282.

55. Soderberg KA, Payne GW, Sato A, Medzhitov R, Segal SS, Iwasaki A. Innate control of adaptive immunity via remodeling of lymph node feed arteriole. Proc Natl Acad Sci U S A. 2005;102(45):16315-16320.

56. Chyou $\mathrm{S}$, et al. Coordinated regulation of lymph node vascular-stromal growth first by CD $11 \mathrm{c}^{+}$ cells and then by T and B cells. J Immunol. 2011; 187(11):5558-5567.

57. Chyou S, et al. Fibroblast-type reticular stromal cells regulate the lymph node vasculature. J Immunol. 2008;181(6):3887-3896.

58. Guarda G, et al. L-selectin-negative CCR7- effector and memory $\mathrm{CD}^{+} \mathrm{T}$ cells enter reactive lymph nodes and kill dendritic cells. Nat Immunol. 2007; 8(7):743-752

59. Martin-Fontecha A, et al. Induced recruitment of NK cells to lymph nodes provides IFN- $\gamma$ for T(H) 1 priming. Nat Immunol. 2004;5(12):1260-1265.

60. Chen Q, et al. Fever-range thermal stress promotes lymphocyte trafficking across high endothelial venules via an interleukin 6 trans-signaling mechanism. Nat Immunol. 2006;7(12):1299-1308.

61. Yoneyama $\mathrm{H}$, et al. Evidence for recruitment of plasmacytoid dendritic cell precursors to inflamed lymph nodes through high endothelial venules. Int Immunol. 2004;16(7):915-928.

62. Takedachi M, et al. CD73-generated adenosine restricts lymphocyte migration into draining lymph nodes. J Immunol. 2008;180(9):6288-6296.

63. Walch JM, et al. Cognate antigen directs $\mathrm{CD}^{+} \mathrm{T}$ cell migration to vascularized transplants. J Clin Invest. 2013;123(6):2663-2671.

64. Kokkonen TS, Augustin MT, Makinen JM, Kok- 
konen J, Karttunen TJ. High endothelial venules of the lymph nodes express Fas ligand.J Histochem Cytochem. 2004;52(5):693-699.

65. Odermatt B, Eppler M, Leist TP, Hengartner H, Zinkernagel RM. Virus-triggered acquired immunodeficiency by cytotoxic T-cell-dependent destruction of antigen-presenting cells and lymph follicle structure. Proc Natl Acad Sci U S A. 1991;88(18):8252-8256.

66. Scandella E, et al. Restoration of lymphoid organ integrity through the interaction of lymphoid tissue-inducer cells with stroma of the T cell zone. Nat Immunol. 2008;9(6):667-675.

67. Soudja SM, et al. Disrupted lymph node and splenic stroma in mice with induced inflammatory melanomas is associated with impaired recruitment of T and dendritic cells. PLoS One. 2011;6(7):e22639.

68. Graw F, Regoes RR. Influence of the fibroblastic reticular network on cell-cell interactions in lymphoid organs. PLoS Comput Biol. 2012;8(3):e1002436.

69. Gorfu G, et al. Laminin isoforms of lymph nodes and predominant role of $\alpha 5$-laminin(s) in adhesion and migration of blood lymphocytes. J Leukoc Biol. 2008;84(3):701-712.

70 . He F, et al. Improvement in endothelial cell adhesion and retention under physiological shear stress using a laminin-apatite composite layer on titanium. J R Soc Interface. 2013;10(81):20130014.

71. Sixt M, Engelhardt B, Pausch F, Hallmann R, Wendler O, Sorokin LM. Endothelial cell laminin isoforms, laminins 8 and 10, play decisive roles in $\mathrm{T}$ cell recruitment across the blood-brain barrier in experimental autoimmune encephalomyelitis. J Cell Biol. 2001;153(5):933-946.
72. Masocha W, Robertson B, Rottenberg ME, Mhlanga J, Sorokin L, Kristensson K. Cerebral vessel laminins and IFN-gamma define Trypanosoma brucei brucei penetration of the blood-brain barrier. J Clin Invest. 2004;114(5):689-694.

73. Wondimu $Z$, et al. An endothelial laminin isoform, laminin 8 ( $\alpha 4 \beta 1 \gamma 1)$, is secreted by blood neutrophils, promotes neutrophil migration and extravasation, and protects neutrophils from apoptosis. Blood. 2004;104(6):1859-1866.

74. Tashiro K, et al. The RGD containing site of the mouse laminin A chain is active for cell attachment, spreading, migration and neurite outgrowth. J Cell Physiol. 1991;146(3):451-459.

75. Nishiuchi $\mathrm{R}$, et al. Characterization of the ligand-binding specificities of integrin $\alpha 3 \beta 1$ and $\alpha 6 \beta 1$ using a panel of purified laminin isoforms containing distinct $\alpha$ chains. J Biochem. 2003;134(4):497-504.

76. Kikkawa Y, Sanzen N, Fujiwara H, Sonnenberg A, Sekiguchi K. Integrin binding specificity of laminin-10/11: laminin-10/11 are recognized by $\alpha 3 \beta 1, \alpha 6 \beta 1$, and $\alpha 6 \beta 4$ integrins. J Cell Sci. 2000; 113(pt 5):869-876

77. Voisin MB, Probstl D, Nourshargh S. Venular basement membranes ubiquitously express matrix protein low-expression regions: characterization in multiple tissues and remodeling during inflammation. Am J Pathol. 2010;176(1):482-495.

78. Kenne E, Soehnlein O, Genove G, Rotzius P, Eriksson EE, Lindbom L. Immune cell recruitment to inflammatory loci is impaired in mice deficient in basement membrane protein laminin $\alpha 4$. J Lenkoc Biol. 2010;88(3):523-528.
79. Li J, et al. Overexpression of laminin-8 in human dermal microvascular endothelial cells promotes angiogenesis-related functions. J Invest Dermatol. 2006;126(2):432-440.

80. Gonzalez AM, et al. Complex interactions between the laminin $\alpha 4$ subunit and integrins regulate endothelial cell behavior in vitro and angiogenesis in vivo. Proc Natl Acad Sci U S A. 2002; 99(25):16075-16080.

81. Grubin CE, Kovats S, deRoos P, Rudensky AY. Deficient positive selection of CD4 $\mathrm{T}$ cells in mice displaying altered repertoires of MHC class II-bound self-peptides. Immunity. 1997;7(2):197-208.

82. Corry RJ, Winn HJ, Russell PS. Primarily vascularized allografts of hearts in mice. The role of $\mathrm{H}-2 \mathrm{D}$, $\mathrm{H}-2 \mathrm{~K}$, and non-H-2 antigens in rejection. Transplantation. 1973;16(4):343-350.

83. Cooper JD, et al. A working formulation for the standardization of nomenclature and for clinical staging of chronic dysfunction in lung allografts. International Society for Heart and Lung Transplantation. J Heart Lung Transplant. 1993;12(5):713-716.

84. Nagano H, Mitchell RN, Taylor MK, Hasegawa S, Tilney NL, Libby P. Interferon- $\gamma$ deficiency prevents coronary arteriosclerosis but not myocardial rejection in transplanted mouse hearts. J Clin Invest. 1997;100(3):550-557.

85. Shimizu K, Schonbeck U, Mach F, Libby P, Mitchell RN. Host CD40 ligand deficiency induces longterm allograft survival and donor-specific tolerance in mouse cardiac transplantation but does not prevent graft arteriosclerosis. J Immunol. 2000; 165(6):3506-3518. 